\title{
Synthesis of Pyrrolo-isoquinolines Related to the Lamellarins Using Silver-Catalyzed Cycloisomerization/Dipolar Cycloaddition
}

\author{
Shun Su and John A. Porco, Jr.* \\ Department of Chemistry and Center for Chemical Methodology and Library Development (CMLD-BU), Boston \\ University, 590 Commonwealth Avenue, Boston, Massachusetts 02215

\section{Supporting Information}

\section{Table of Contents}

I. General Experimental Procedures..................................................

II. Preparation of Alkynyl $N$-benzylidene Glycinates.....................................SI-3

III. Reaction Screening................................................................. SI-4

IV. Synthesis of Pyrrolo-isoquinolines...................................................... SI-5

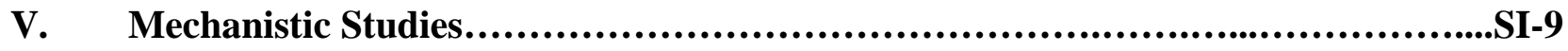

VI. X-ray Crystallographic Data.....................................................SI-14

VII. Select NMR Spectra................................................................... 


\section{General Experimental Procedures}

${ }^{1} \mathrm{H}$ NMR spectra were recorded on a Varian Mercury $400 \mathrm{MHz}$ spectrometer at ambient temperature in $\mathrm{CDCl}_{3}$ unless otherwise stated. ${ }^{13} \mathrm{C}$ NMR spectra were recorded at $100 \mathrm{MHz}$ spectrometer (unless otherwise stated) at ambient temperature. Chemical shifts are reported in parts per million relative to chloroform $\left({ }^{1} \mathrm{H}, \delta 7.24 ;{ }^{13} \mathrm{C}, \delta 77.23\right)$. Data for ${ }^{1} \mathrm{H} \mathrm{NMR}$ are reported as follows: chemical shift, integration, multiplicity $(\operatorname{app}=$ apparent, par obsc $=$ partially obscure, ovrlp = overlapping, $\mathrm{s}=$ singlet, $\mathrm{d}=$ doublet, $\mathrm{t}=$ triplet, $\mathrm{q}=$ quartet, $\mathrm{m}=$ multiplet) and coupling constants. All ${ }^{13} \mathrm{C}$ NMR spectra were recorded with complete proton decoupling. High-resolution mass spectra were obtained in the Boston University Chemical Instrumentation Center using a Waters Q-TOF mass spectrometer. Melting points were recorded on a Mel-Temp (Laboratory Devices). Infrared spectra were recorded on a Nicolet Nexus 670 FT-IR. HPLC-ELSD-MS analysis was performed on a Waters HPLC-MS system equipped with a Waters 600 HPLC pump, Waters 2996 photodiode array detector, Micromass ZQ Quadrupole Mass Spectrometer, and Sedere Sedex 75 ELS detector (SunFire ${ }^{\mathrm{TM}} \mathrm{C}_{18}$ column, $5 \mu \mathrm{m}, 4.6 \times 50 \mathrm{~mm}$ ). Reaction screening was carried out in MiniBlocks ${ }^{\circledR}$ (Mettler Toledo AutoChem, Inc). Analytical thin layer chromatography was performed on $0.25 \mathrm{~mm}$ silica gel $60-\mathrm{F}$ plates and $150 \mu \mathrm{m} \mathrm{C}_{18}$ silica TLC plates (Sorbent Technologies). Flash chromatography was performed using a CombiFlash ${ }^{\circledR}$ Sq16X system (Teledyne ISCO) using normal phase RediSep ${ }^{\mathrm{TM}}$ flash columns. Sep-Pak ${ }^{\circledR}$ Vac 12 cc (2 g) silica cartridges were obtained from Waters, Inc. (Part No. WAT 036920). All reagents were used as supplied by Sigma-Aldrich, Lancaster Synthesis, and Strem Chemicals. Methylene chloride, tetrahydrofuran, methanol, and benzene were purified by passing through two packed columns of neutral alumina (Innovative Technology, Newburyport, $\mathrm{MA}$ ). $\mathrm{CDCl}_{3}$ and toluene- $\mathrm{d}_{8}$ used for compound characterization were neutralized using basic alumina. The Arthur ${ }^{\mathrm{TM}}$ Suite Reaction Planner (Symyx Technologies, Inc.) was used for experimental procedure planning. 


\section{Preparation of Alkynyl N-benzylidene Glycinates}
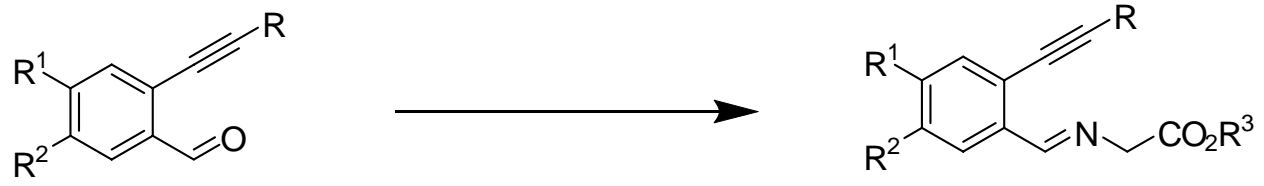

\section{Representative Procedure:}

A) To a round bottom flask was added the appropriate alkynylbenzaldehyde (0.2 mmol), Me-Gly$\mathrm{NH}_{2} . \mathrm{HCl}(50 \mathrm{mg}, 0.4 \mathrm{mmol}), \mathrm{NaHCO}_{3}(84 \mathrm{mg}, 1.0 \mathrm{mmol}), \mathrm{MgSO}_{4}(200 \mathrm{mg})$, and $\mathrm{CH}_{2} \mathrm{Cl}_{2}(3 \mathrm{~mL})$ under an argon atmosphere. The reaction was allowed to stir at $\mathrm{rt}$ overnight, filtered, and concentrated to provide the desired alkynyl imine $N$-benzylidene glycinate which was used in the cycloisomerization/dipolar-cycloaddition step without further purification.

B) For the synthesis of alkynyl imine 16, instead of $\mathrm{NaHCO}_{3}, \mathrm{Et}_{3} \mathrm{~N}$ (3.0 equiv.) was used as base. Upon completion of the reaction, the reaction mixture was concentrated, dissolved in EtOAc, and filtered to remove $\mathrm{Et}_{3} \mathrm{~N}$.HCl. Concentration of the filtrate provided $\mathbf{1 6}$ without contamination of $\mathrm{Et}_{3} \mathrm{~N} . \mathrm{HCl}$.

C) For alkynyl imines 14 and 17, Boc-Gly- $\mathrm{NH}_{2}$ was used without addition of base. 


\section{Reaction Screening}

General procedure for reaction screening: To a MiniBlock ${ }^{\circledR}$ reaction block was added alkynyl imine 6 (6.0 mg, $0.02 \mathrm{mmol})$, DMAD $7(5.7 \mathrm{mg}, 0.04 \mathrm{mmol})$, DIEA $(7.0 \mu \mathrm{L}, 0.04 \mathrm{mmol})$, and DCE $(0.5 \mathrm{~mL})$. Next, $8 \mathrm{mg}$ of a catalyst $/ 4 \AA$ molecular sieve mixture ${ }^{1}(1.25 \mathrm{mmol}$ catalyst $/ \mathrm{g}$ molecular sieves, $0.01 \mathrm{~mol}$ ) was added. Reactions were allowed to stir in the air for $5 \mathrm{~h}$ at $50^{\circ} \mathrm{C}$, cooled to rt, and finally filtered through a silica gel plug eluting with EtOAc $(2 \mathrm{~mL})$. The solution was concentrated and directly subjected to ${ }^{1} \mathrm{H}$ NMR analysis.

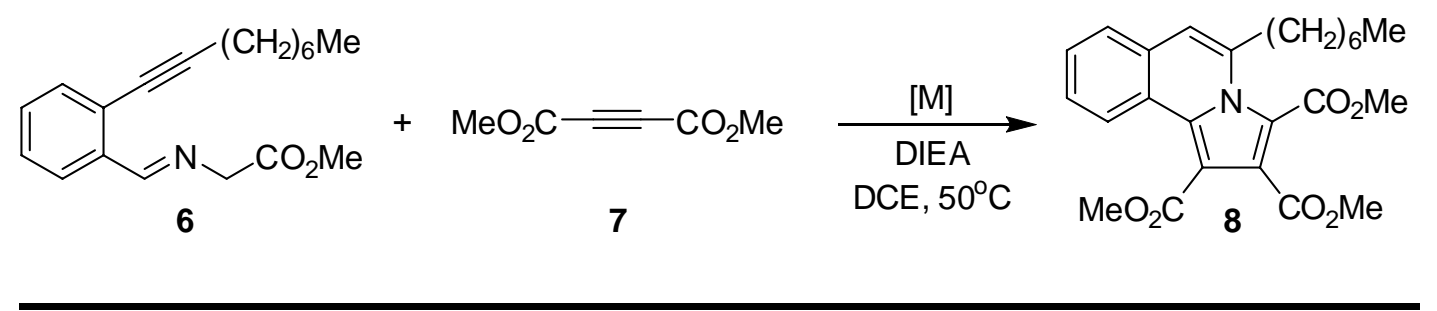

\begin{tabular}{llllll}
\hline Catalysts & & & & & \\
$\mathrm{PPh}_{3} \mathrm{AuCl}$ & $\mathrm{Au}(\mathrm{OAC})_{3}$ & $\mathrm{AgSbF}_{6}$ & $\mathrm{AgOTf}$ & $\mathrm{Cu}(\mathrm{MeCN})_{4} \mathrm{PF}_{6}$ & $\mathrm{Cu}(\mathrm{OTf})_{2}$ \\
$\mathrm{Pd}(\mathrm{OAC})_{2}$ & $\mathrm{PtCl}_{2}$ & $\mathrm{PtCl}_{4}$ & $\mathrm{Rh}(\mathrm{COD})_{2} \mathrm{BF}_{4}$ & $\ln (\mathrm{OTf})_{3}$ & \\
\hline
\end{tabular}

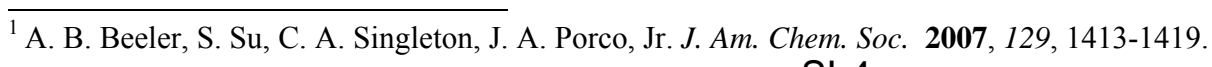




\section{Synthesis of Pyrrolo-isoquinolines}

Representative procedure for the synthesis of lamellarin-type molecules: To a round bottom flask was added alkynyl imine 6 ( $0.15 \mathrm{mmol}$, crude product from corresponding aldehyde), DMAD (25.6 mg, $0.18 \mathrm{mmol})$, DTBMP (31 mg, $0.15 \mathrm{mmol})$, and toluene (5 mL). AgOTf (3.9 mg, $0.015 \mathrm{mmol}$ ) was next added and the reaction mixture was heated to $60{ }^{\circ} \mathrm{C}$, stirred for $6 \mathrm{~h}$ in the air, and finally cooled to rt. Filtration through a silica gel plug $(3.0 \mathrm{~g})$ using EtOAc $(10 \mathrm{~mL})$ followed by concentration provided a crude product which was purified by flash chromatography $\left(\mathrm{SiO}_{2}, 0\right.$ $25 \%$ EtOAc in hexanes) to provide the desired pyrrolo-isoquinoline 8 as a yellow solid (47 $\mathrm{mg}$, $72 \%)$.

For the synthesis of 33-37, 2.5 equiv. of alkynes were used. For compounds 35 and 37, 5 mol\% of $\mathrm{Yb}(\mathrm{OTf})_{3}$ was added upon addition of AgOTf.

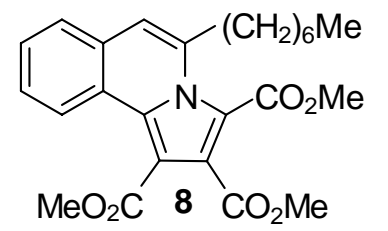

Pyrrolo-isoquinoline 8: Yellow solid m.p. 79.0-81.0 ${ }^{\circ} \mathrm{C} ;{ }^{1} \mathrm{H}$ NMR (400 $\left.\mathrm{MHz}, \mathrm{CDCl}_{3}\right) \delta 8.39(\mathrm{~m}, 1 \mathrm{H}), 7.58(\mathrm{~m}, 1 \mathrm{H}), 7.49(\mathrm{~m}, 2 \mathrm{H}), 6.86(\mathrm{~s}, 1 \mathrm{H})$, $4.00(\mathrm{~s}, 3 \mathrm{H}), 3.97(\mathrm{~s}, 3 \mathrm{H}), 3.90(\mathrm{~s}, 3 \mathrm{H}), 2.89(\mathrm{t}, 2 \mathrm{H}, J=7.6 \mathrm{~Hz}), 1.71(\mathrm{~m}$, $2 \mathrm{H}), 1.35(\mathrm{~m}, 8 \mathrm{H}), 0.89(\mathrm{t}, 3 \mathrm{H}, J=6.6 \mathrm{~Hz}) ;{ }^{13} \mathrm{C} \mathrm{NMR}\left(100 \mathrm{MHz}, \mathrm{CDCl}_{3}\right) \delta$ $167.1,164.4,163.7,137.3,131.3,128.8,128.3,127.8,126.7,124.2,123.7$, $121.0,120.7,114.1,109.5,53.4,52.9,52.6,32.4,31.9,29.4,29.2,27.2,22.8,14.3$; IR (thin film) vmax 2951, 2931, 2856, 1724, 1548, 1511, 1462, 1360, 1251, 1222, 1179, 1119, 1075, 1049, 1025, 982, 944, $837 \mathrm{~cm}^{-1}$; HRMS (ESI+) [M+Na] [' calculated for $\mathrm{C}_{25} \mathrm{H}_{29} \mathrm{NO}_{6} \mathrm{Na}$ : 462.1893, found: 462.1933.

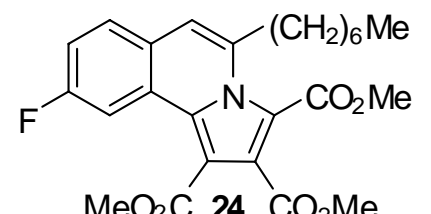

Pyrrolo-isoquinoline 24: Yellow solid m.p. 92.0-93.5 ${ }^{\circ} \mathrm{C} ;{ }^{1} \mathrm{H}$ NMR $\left(400 \mathrm{MHz}, \mathrm{CDCl}_{3}\right) \delta 8.19(\mathrm{dd}, 1 \mathrm{H}, J=2.2 \mathrm{~Hz}, J=10.9 \mathrm{~Hz}), 7.56(\mathrm{dd}, 1 \mathrm{H}$, $J=5.8 \mathrm{~Hz}, J=8.6 \mathrm{~Hz}), 7.23(\mathrm{~m}, 1 \mathrm{H}), 6.83(\mathrm{~s}, 1 \mathrm{H}), 4.00(\mathrm{~s}, 3 \mathrm{H}), 3.97(\mathrm{~s}$, $3 \mathrm{H}), 3.90(\mathrm{~s}, 3 \mathrm{H}), 2.87(\mathrm{t}, 2 \mathrm{H}, J=7.6 \mathrm{~Hz}), 1.69(\mathrm{~m}, 2 \mathrm{H}), 1.28(\mathrm{~m}, 8 \mathrm{H})$, $0.88(\mathrm{t}, 3 \mathrm{H}, J=6.6 \mathrm{~Hz}) ;{ }^{13} \mathrm{C}$ NMR $\left(100 \mathrm{MHz}, \mathrm{CDCl}_{3}\right) \delta 166.6,164.3$, $163.4,162.9,160.5,136.7,136.7,130.7,128.85,128.76,125.5,125.1,125.0,121.6,120.9,117.2$, $116.9,113.4,110.2,110.0,109.9,53.4,53.0,52.6,32.4,31.9,29.4,29.2,27.2,22.8,14.2$; IR (thin film) vmax 2953, 2923, 2873, 2858, 1724, 1615, 1555, 1515, 1452, 1434, 1338, 1285, 1248, 1229 , 1197, 1175, 1112, 1100, 1047, 1033, 921, $856 \mathrm{~cm}^{-1}$; HRMS (ESI+) $[\mathrm{M}+\mathrm{Na}]^{+}$calculated for $\mathrm{C}_{25} \mathrm{H}_{28} \mathrm{FNO}_{6} \mathrm{Na}: 480.1798$, found: 480.1805 .

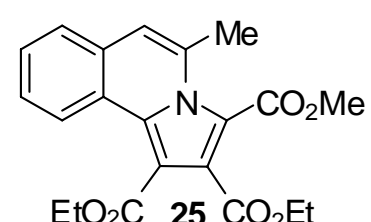

Pyrrolo-isoquinoline 25: Yellow solid m.p. 101.0-102.5 ${ }^{\circ} \mathrm{C} ;{ }^{1} \mathrm{H}$ NMR $(400$ $\left.\mathrm{MHz} \mathrm{CDCl}_{3}\right) \delta 8.38(\mathrm{~m}, 1 \mathrm{H}), 7.48(\mathrm{~m}, 3 \mathrm{H}), 6.78(\mathrm{~s}, 1 \mathrm{H}), 4.48(\mathrm{q}, 2 \mathrm{H}, J=$ $7.2 \mathrm{~Hz}), 4.36(\mathrm{q}, 2 \mathrm{H}, J=7.2 \mathrm{~Hz}), 3.97(\mathrm{~s}, 3 \mathrm{H}), 2.55(\mathrm{~s}, 3 \mathrm{H}), 1.42(\mathrm{t}, 3 \mathrm{H}, J=$ $7.2 \mathrm{~Hz}), 1.37(\mathrm{t}, 3 \mathrm{H}, J=7.2 \mathrm{~Hz}) ;{ }^{13} \mathrm{C} \mathrm{NMR}\left(100 \mathrm{MHz}, \mathrm{CDCl}_{3}\right) \delta 166.7$, $163.8,132.8,130.6,128.7,128.2,127.7,126.5,124.1,123.8,120.8,120.5$, $115.9,110.0,61.9,61.4,53.3,20.2,14.4,14.3$; IR (thin film) vmax 2990, 2967, 2954, 2913, 1721, 1563, 1509, 1483, 1465, 1455, 1442, 1399, 1385, 1355, 1334, 1250, 1217, 1194, 1127, 1074, 1050, $1027,864,847,742 \mathrm{~cm}^{-1}$; HRMS (ESI+) $[\mathrm{M}+\mathrm{Na}]^{+}$calculated for $\mathrm{C}_{21} \mathrm{H}_{21} \mathrm{NO}_{6} \mathrm{Na}$ : 406.1267, found: 406.1273 . 


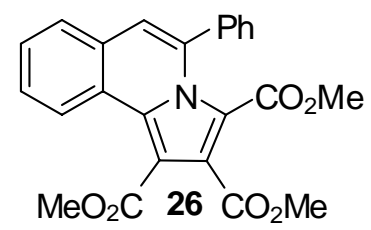

Pyrrolo-isoquinoline 26: Yellow solid m.p. $147.0-148.5{ }^{\circ} \mathrm{C} ;{ }^{1} \mathrm{H}$ NMR $(400$ $\left.\mathrm{MHz}, \mathrm{CDCl}_{3}\right) \delta 8.80(\mathrm{~m}, 1 \mathrm{H}), 7.64(\mathrm{~m}, 1 \mathrm{H}), 7.49(\mathrm{~m}, 7 \mathrm{H}), 6.96(\mathrm{~s}, 1 \mathrm{H})$, $3.99(\mathrm{~s}, 3 \mathrm{H}), 3.88(\mathrm{~s}, 3 \mathrm{H}), 3.23(\mathrm{~s}, 3 \mathrm{H}) ;{ }^{13} \mathrm{C}$ NMR $\left(100 \mathrm{MHz}, \mathrm{CDCl}_{3}\right) \delta$ $166.2,164.9,161.1,136.6,136.2,133.1,129.5,129.4,129.1,128.9,128.4$, $127.6,127.4,125.2,124.2,123.9,120.6,118.2,109.1,52.8,52.7,52.2 ;$ IR (thin film) vmax 3060, 3034, 2951, 1737, 1719, 1706, 1537, 1509, 1496, 1475, 1433, 1387, 1229, $1163,1121,1087,768 \mathrm{~cm}^{-1}$; HRMS $(\mathrm{ESI}+)[\mathrm{M}+\mathrm{H}]^{+}$calculated for $\mathrm{C}_{24} \mathrm{H}_{20} \mathrm{NO}_{6}: 418.1291$, found: 418.1300 .

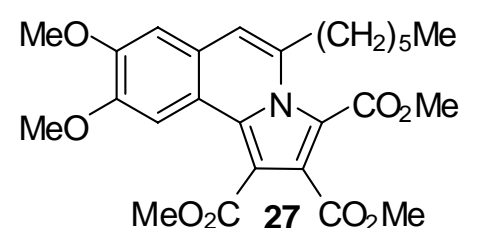

Pyrrolo-isoquinoline 27: Yellow solid m.p. 109.0-111.0 ${ }^{\circ} \mathrm{C} ;{ }^{1} \mathrm{H}$ NMR $\delta 8.52(\mathrm{~s}, 1 \mathrm{H}), 6.97(\mathrm{~s}, 1 \mathrm{H}), 6.86(\mathrm{~s}, 1 \mathrm{H}), 4.01(\mathrm{~s}, 3 \mathrm{H}), 3.98(\mathrm{~s}$, $3 \mathrm{H}), 3.92(\mathrm{~s}, 6 \mathrm{H}), 3.91(\mathrm{~s}, 3 \mathrm{H}), 2.90(\mathrm{t}, 2 \mathrm{H}, J=7.6 \mathrm{~Hz}), 1.64(\mathrm{~m}, 2 \mathrm{H})$, $1.31(\mathrm{~m}, 6 \mathrm{H}), 0.87(\mathrm{t}, 3 \mathrm{H}, J=6.5 \mathrm{~Hz}) ;{ }^{13} \mathrm{C}$ NMR $\left(100 \mathrm{MHz}, \mathrm{CDCl}_{3}\right) \delta$ $166.2,165.6,162.7,150.6,149.4,136.3,134.0,125.4,125.0,118.6$, 118.1, 114.2, 106.78, 106.76, 106.6, 56.3, 56.1, 53.0, 52.6, 52.5, 33.1, 31.8, 29.1, 27.2, 22.7, 14.2; IR (thin film) vmax 3000, 2955, 2925, 2857, 1724, 1615, 1547, 1502, 1469, 1442, 1378, 1258, $1211,1159,1123,1097,1080,1050,978,865 \mathrm{~cm}^{-1}$; HRMS (ESI+) $[\mathrm{M}+\mathrm{H}]^{+}$calculated for $\mathrm{C}_{26} \mathrm{H}_{32} \mathrm{NO}_{8}$ : 486.2198, found: 486.2132 .

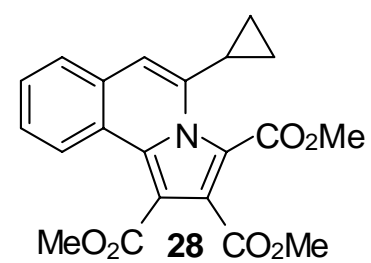

Pyrrolo-isoquinoline 28: Yellow solid m.p. 106.0-108.0 ${ }^{\circ} \mathrm{C} ;{ }^{1} \mathrm{H}$ NMR $(400$ $\left.\mathrm{MHz}, \mathrm{CDCl}_{3}\right) \delta 8.40(\mathrm{~m}, 1 \mathrm{H}), 7.56(\mathrm{~m}, 1 \mathrm{H}), 7.48(\mathrm{~m}, 2 \mathrm{H}), 6.92(\mathrm{~s}, 1 \mathrm{H})$, $4.00(\mathrm{~s}, 3 \mathrm{H}), 3.96(\mathrm{~s}, 3 \mathrm{H}), 3.90(\mathrm{~s}, 3 \mathrm{H}), 2.28(\mathrm{~m}, 1 \mathrm{H}), 0.96(\mathrm{~m}, 2 \mathrm{H}), 0.75(\mathrm{~m}$, $2 \mathrm{H}) ;{ }^{13} \mathrm{C}$ NMR $\left(100 \mathrm{MHz}, \mathrm{CDCl}_{3}\right) \delta 167.2,164.4,163.7,137.9,130.6$, $128.7,128.2,128.1,126.8,124.2,123.9,121.1,120.5,115.5,109.7,53.2$, $52.9,52.6,14.7,8.1$; IR (thin film) vmax 3076, 3002, 2954, 1733, 1717, $1535,1507,1474,1463,1432,1387,1372,1254,1220,1178,1119,1080,1022,1054,984,882$ $\mathrm{cm}^{-1}$; HRMS (ESI + ) $[\mathrm{M}+\mathrm{H}]^{+}$calculated for $\mathrm{C}_{21} \mathrm{H}_{20} \mathrm{NO}_{6}$ : 382.1291 , found: 382.1310 .

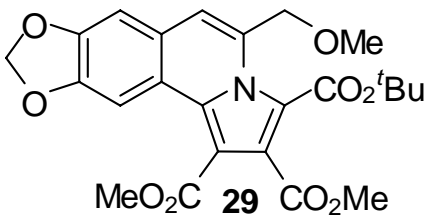

Pyrrolo-isoquinoline 29: White solid m.p. $138.5-140.0{ }^{\circ} \mathrm{C} ;{ }^{1} \mathrm{H}$ NMR $\left(400 \mathrm{MHz}, \mathrm{CDCl}_{3}\right) \delta 8.55(\mathrm{~s}, 1 \mathrm{H}), 7.09(\mathrm{~s}, 1 \mathrm{H}), 7.00(\mathrm{~s}, 1 \mathrm{H}), 6.07(\mathrm{~s}$, $2 \mathrm{H}), 4.76(\mathrm{~s}, 2 \mathrm{H}), 3.93(\mathrm{~s}, 3 \mathrm{H}), 3.92(\mathrm{~s}, 3 \mathrm{H}), 3.23(\mathrm{~s}, 3 \mathrm{H}), 1.59(\mathrm{~s}, 9 \mathrm{H})$; ${ }^{13} \mathrm{C}$ NMR $\left(100 \mathrm{MHz}, \mathrm{CDCl}_{3}\right) \delta 166.2,165.5,159.9,149.1,148.6$, $134.4,132.3,126.9,126.0,120.2,119.4,115.8,106.5,104.8,104.7$, $101.9,83.0,72.0,58.0,52.6,52.4,28.1$; IR (thin film) vmax 2996, 2979, 2954, 2904, 2830, 1731, $1705,1534,1505,1473,1442,1389,1379,1355,1318,1227,1145,1089,1037,941,859,843 \mathrm{~cm}^{-}$ ${ }^{1}$; HRMS (ESI+) [M+Na] $]^{+}$calculated for $\mathrm{C}_{24} \mathrm{H}_{25} \mathrm{NO}_{9} \mathrm{Na}$ : 494.1427, found: 494.1469.

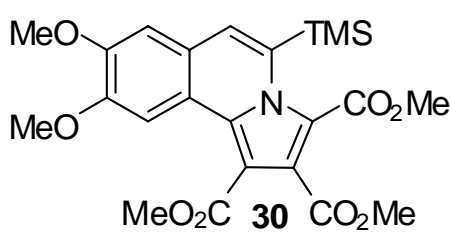

Pyrrolo-isoquinoline 30: Yellow solid m.p. $166.0-168.5{ }^{\circ} \mathrm{C} ;{ }^{1} \mathrm{H}$ NMR $\left(400 \mathrm{MHz}, \mathrm{CDCl}_{3}\right) \delta 8.77(\mathrm{~s}, 1 \mathrm{H}), 7.03(\mathrm{~s}, 1 \mathrm{H}), 6.66(\mathrm{~s}, 1 \mathrm{H})$, $3.68(\mathrm{~s}, 3 \mathrm{H}), 3.63(\mathrm{~s}, 3 \mathrm{H}), 3.56(\mathrm{~s}, 3 \mathrm{H}), 3.50(\mathrm{~s}, 3 \mathrm{H}), 3.48(\mathrm{~s}, 3 \mathrm{H})$, $0.00(\mathrm{~s}, 9 \mathrm{H}) ;{ }^{13} \mathrm{C}$ NMR $\left(100 \mathrm{MHz}, \mathrm{CDCl}_{3}\right) \delta 167.1,164.7,161.7$, $151.1,150.1,139.3,137.2,131.1,127.6,125.0,119.7,117.4,108.3$, 106.8, 105.9, 56.5, 56.2, 52.7, 52.2, 52.2, 2.0; IR (thin film) vmax 3151, 3114, 2950, 2904, 2844, $1759,1707,1690,1616,1548,1477,1441,1412,1399,1375,1336,1246,1214,1140,1089,1051$, $1030,1002,938,877,849,810,795 \mathrm{~cm}^{-1}$; HRMS (ESI+) $[\mathrm{M}+\mathrm{Na}]^{+}$calculated for $\mathrm{C}_{23} \mathrm{H}_{27} \mathrm{NO}_{8} \mathrm{SiNa}$ : 496.1404, found: 496.1366 . 


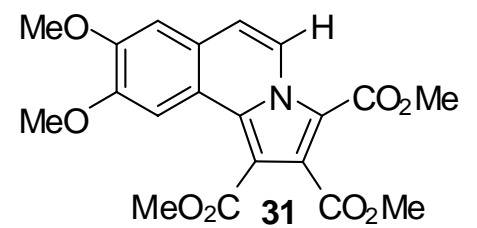

Pyrrolo-isoquinoline 31: Pale yellow solid m.p. $190.5-192.0{ }^{\circ} \mathrm{C} ;{ }^{1} \mathrm{H}$ NMR (400 MHz, $\left.\mathrm{CDCl}_{3}\right) \delta 9.32(\mathrm{~s}, 1 \mathrm{H}), 9.27(\mathrm{~d}, 1 \mathrm{H}, J=7.5 \mathrm{~Hz}), 7.26$ $(\mathrm{s}, 1 \mathrm{H}), 7.14(\mathrm{~d}, 1 \mathrm{H}, J=7.5 \mathrm{~Hz}), 7.07(\mathrm{~s}, 1 \mathrm{H}), 4.08(\mathrm{~s}, 3 \mathrm{H}), 4.02(\mathrm{~s}$, $3 \mathrm{H}), 3.97(\mathrm{~s}, 3 \mathrm{H}), 3.91(\mathrm{~s}, 3 \mathrm{H}), 3.90(\mathrm{~s}, 3 \mathrm{H}) ;{ }^{13} \mathrm{C}$ NMR $(100 \mathrm{MHz}$, $\left.\mathrm{CDCl}_{3}\right) \delta 167.0,164.4,160.6,151.2,149.8,135.3,131.1,125.8$, $122.8,119.5,115.5,112.8,108.9,107.0,106.0,56.5,56.2,52.8,52.3,52.2$; IR (thin film) vmax 3070, 3007, 2953, 2851, 1741, 1673, 1633, 1489, 1454, 1397, 1365, 1321, 1290, 1243, 1195, 1033, $833,758 \mathrm{~cm}^{-1}$; HRMS (ESI + ) $[\mathrm{M}+\mathrm{Na}]^{+}$calculated for $\mathrm{C}_{20} \mathrm{H}_{19} \mathrm{NO}_{8} \mathrm{Na}$ : 424.1008 , found: 424.0948 .

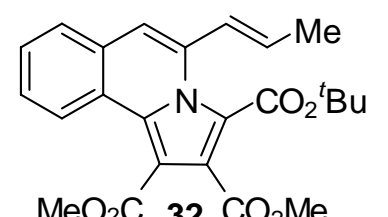

Pyrrolo-isoquinoline 32: Yellow solid m.p. 107.5-109.0 ${ }^{\circ} \mathrm{C} ;{ }^{1} \mathrm{H}$ NMR $(400$ $\left.\mathrm{MHz}, \mathrm{CDCl}_{3}\right) \delta 8.63(\mathrm{~m}, 1 \mathrm{H}), 7.61(\mathrm{~m}, 1 \mathrm{H}), 7.49(\mathrm{~m}, 2 \mathrm{H}), 7.01(\mathrm{~s}, 1 \mathrm{H})$, $6.47(\mathrm{~d}, 1 \mathrm{H}, J=16.6 \mathrm{~Hz}), 6.39(\mathrm{~m}, 1 \mathrm{H}), 3.98(\mathrm{~s}, 3 \mathrm{H}), 3.91(\mathrm{~s}, 3 \mathrm{H}), 1.93(\mathrm{~d}$, $3 \mathrm{H}, J=6.3 \mathrm{~Hz}), 1.60(\mathrm{~s}, 9 \mathrm{H}) ;{ }^{13} \mathrm{C} \mathrm{NMR}\left(100 \mathrm{MHz}, \mathrm{CDCl}_{3}\right) \delta 166.6,164.9$, $160.9,135.4,131.6,131.2,129.3,128.4,127.9,127.0,125.7,124.9,124.1$, $122.1,121.8,114.7,108.9,83.5,52.7,52.4,28.1,18.9$; IR (thin film) vmax 3028, 2982, 2950, $2857,1733,1721,1533,1511,1471,1437,1394,1368,1358,1254,1220,1181,1118,1075,1047$, 991, 967, 840, $751 \mathrm{~cm}^{-1}$; HRMS (ESI+) $[\mathrm{M}+\mathrm{H}]^{+}$calculated for $\mathrm{C}_{24} \mathrm{H}_{26} \mathrm{NO}_{6}: 424.1760$, found: 424.1801 .

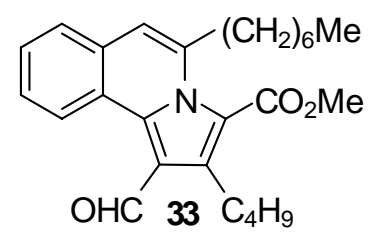

Pyrrolo-isoquinoline 33: pale yellow oil; ${ }^{1} \mathrm{H} \mathrm{NMR}\left(400 \mathrm{MHz}, \mathrm{CDCl}_{3}\right) \delta$ $10.46(\mathrm{~s}, 1 \mathrm{H}), 9.38(\mathrm{dd}, 1 \mathrm{H}, J=3.2 \mathrm{~Hz}, J=6.0 \mathrm{~Hz}), 7.67(\mathrm{dd}, 1 \mathrm{H}, J=3.4 \mathrm{~Hz}$, $\mathrm{J}=5.9 \mathrm{~Hz}), 7.60(\mathrm{~m}, 2 \mathrm{H}), 7.02(\mathrm{~s}, 1 \mathrm{H}), 3.96(\mathrm{~s}, 3 \mathrm{H}), 3.07(\mathrm{t}, 2 \mathrm{H}, J=7.6 \mathrm{~Hz})$, $2.92(\mathrm{t}, 2 \mathrm{H}, J=8.0 \mathrm{~Hz}), 1.69(\mathrm{~m}, 4 \mathrm{H}), 1.36(\mathrm{~m}, 10 \mathrm{H}), 0.89(\mathrm{~m}, 6 \mathrm{H}) ;{ }^{13} \mathrm{C}$ NMR $\left(100 \mathrm{MHz}, \mathrm{CDCl}_{3}\right) \delta 185.0,163.5,139.3,138.5,138.1,130.7,129.3$, $127.4,127.2$, 126.3, 123.9, 118.9, 116.2, 114.4, 52.3, 33.7, 32.2, 32.1, 31.9, 29.5, 29.2, 27.5, 25.2, 22.8, 22.7, 14.2; IR (thin film) vmax 3068, 2955, 2924, 2857, 2802, 2770, 1711, 1661, 1521, 1496, 1458, 1436, 1395, 1221, 1153, 1170, 1109, 1046, $826 \mathrm{~cm}^{-1}$; HRMS (ESI+) $[\mathrm{M}+\mathrm{Na}]^{+}$calculated for $\mathrm{C}_{26} \mathrm{H}_{33} \mathrm{NO}_{3} \mathrm{Na}: 430.2358$, found: 430.2366 . In order to determine the regiochemistry of the dipolarcycloaddition, compound $33(5.0 \mathrm{mg}, 0.012 \mathrm{mmol})$ was added to a round bottom flask followed by the addition of $\mathrm{MeOH}(1 \mathrm{~mL})$. The solution was cooled to $0{ }^{\circ} \mathrm{C}$ and $\mathrm{NaBH}_{4}(3.8 \mathrm{mg}, 0.1 \mathrm{mmol})$ was added. The reaction was stirred at $0{ }^{\circ} \mathrm{C}$ for $30 \mathrm{~min}$, warmed to $\mathrm{rt}$, and filtered through silica plug $(2 \mathrm{~g})$ using EtOAc as eluent. Concentration provided the corresponding primary alcohol which was analyzed by NOESY spectroscopy (see pages SI 26-27).

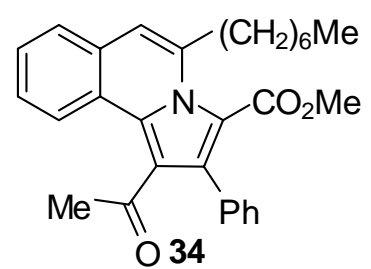

Pyrrolo-isoquinoline 34: Yellow solid m.p. 112.5-114.0 ${ }^{\circ} \mathrm{C} ;{ }^{1} \mathrm{H}$ NMR (400 $\left.\mathrm{MHz}, \mathrm{CDCl}_{3}\right) \delta 8.43(\mathrm{~d}, 1 \mathrm{H}, J=8.2 \mathrm{~Hz}), 7.62(\mathrm{~d}, 1 \mathrm{H}, J=7.7 \mathrm{~Hz}), 7.46(\mathrm{~m}$, $2 \mathrm{H}), 7.39(\mathrm{~m}, 5 \mathrm{H}), 6.92(\mathrm{~s}, 1 \mathrm{H}), 3.61(\mathrm{~s}, 3 \mathrm{H}), 2.95(\mathrm{t}, 2 \mathrm{H}, J=7.6 \mathrm{~Hz}), 2.13$ $(\mathrm{s}, 3 \mathrm{H}), 1.72(\mathrm{~m}, 2 \mathrm{H}), 1.33(\mathrm{~m}, 8 \mathrm{H}), 0.88(\mathrm{t}, 3 \mathrm{H}, J=6.6 \mathrm{~Hz}) ;{ }^{13} \mathrm{C}$ NMR $(100$ $\left.\mathrm{MHz}, \mathrm{CDCl}_{3}\right) \delta 202.4,163.7,137.9,134.1,133.0,131.7,130.3,129.7$, $128.2,128.11,128.06,127.2,126.6,124.5,123.8,120.4,117.6,113.5,52.2$, $33.5,33.2,31.9,29.5,29.2,27.2,22.8,14.3$; IR (thin film) vmax 2950, 2926, 2867, 2852, 1719 , $1675,1536,1511,1468,1457,1443,1292,1246,1224,1178,1162,1112,749 \mathrm{~cm}^{-1}$; HRMS (ESI+) $[\mathrm{M}+\mathrm{H}]^{+}$calculated for $\mathrm{C}_{2} \mathrm{H}_{32} \mathrm{NO}_{3}$ : 442.2382, found: 442.2380. In order to determine the regiochemistry of the dipolar-cycloaddition, compound $34(5.0 \mathrm{mg}, 0.012 \mathrm{mmol})$ was added to a round bottom flask followed by the addition of $\mathrm{MeOH}(1 \mathrm{~mL})$. The solution was cooled to $0{ }^{\circ} \mathrm{C}$ and $\mathrm{NaBH}_{4}(3.8 \mathrm{mg}, 0.1 \mathrm{mmol})$ was added. The reaction was stirred at $0{ }^{\circ} \mathrm{C}$ for $1 \mathrm{~h}$, warmed to $\mathrm{rt}$, and filtered through silica plug $(2 \mathrm{~g})$ using EtOAc as eluant. Concentration provided the corresponding alcohol which was analyzed by NOESY spectroscopy (see page SI-28). 


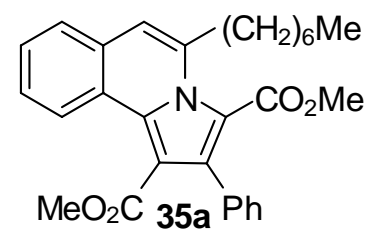

Pyrrolo-isoquinoline 35a: Yellow solid m.p. 94.0-96.0 ${ }^{\circ} \mathrm{C} ;{ }^{1} \mathrm{H}$ NMR $(400$ $\left.\mathrm{MHz}, \mathrm{CDCl}_{3}\right) \delta 8.58(\mathrm{~m}, 1 \mathrm{H}), 7.63(\mathrm{~m}, 1 \mathrm{H}), 7.50(\mathrm{~m}, 2 \mathrm{H}), 7.36(\mathrm{~m}, 5 \mathrm{H})$, $6.92(\mathrm{~s}, 1 \mathrm{H}), 3.65(\mathrm{~s}, 3 \mathrm{H}), 3.61(\mathrm{~s}, 3 \mathrm{H}), 2.95(\mathrm{t}, 2 \mathrm{H}, J=7.6 \mathrm{~Hz}), 1.71(\mathrm{~m}$, $2 \mathrm{H}), 1.32(\mathrm{~m}, 8 \mathrm{H}), 0.88(\mathrm{t}, 3 \mathrm{H}, J=6.8 \mathrm{~Hz}) ;{ }^{13} \mathrm{C}$ NMR $\left(100 \mathrm{MHz}, \mathrm{CDCl}_{3}\right) \delta$ $167.9,163.7,138.1,134.4,134.1,133.1,129.8,129.6,128.1,127.7,127.5$, 127.2, 126.5, 124.8, 123.5, 117.8, 113.4, 110.2, 52.2, 52.0, 33.5, 31.9, 29.5, 29.2, 27.2, 22.8, 14.3; IR (thin film) vmax 3060, 3028, 3004, 2949, 2927, 2867, 2852, 1717, 1604, 1553, 1525, 1445, $1430,1361,1347,1248,1226,1192,1173,1161,1117,1053,1019,749 \mathrm{~cm}^{-1}$; HRMS (ESI+) $[\mathrm{M}+\mathrm{Na}]^{+}$calculated for $\mathrm{C}_{29} \mathrm{H}_{31} \mathrm{NO}_{4} \mathrm{Na}$ : 480.2151, found: 480.2151. In order to determine the regiochemistry of the dipolar-cycloaddition step, compound $35 \mathrm{a}(10 \mathrm{mg}, 0.022 \mathrm{mmol})$ was added to a round bottom flask followed by the addition of THF $(2 \mathrm{~mL})$ under an argon atmosphere. The solution was cooled to $0{ }^{\circ} \mathrm{C}$ and $\mathrm{LiAlH}_{4}(3.8 \mathrm{mg}, 0.1 \mathrm{mmol})$ was added. The reaction was stirred at rt for $2 \mathrm{~h}$, quenched by addition of sat. $\mathrm{NH}_{4} \mathrm{Cl}$, extracted with EtOAc, dried with $\mathrm{Na}_{2} \mathrm{SO}_{4}$, and filtered through a silica gel pad $(2 \mathrm{~g})$ using EtOAc as eluant. Concentration and flash chromatography provided the corresponding alcohol which was analyzed by 2D-NMR spectroscopy (see pages SI 29-30).

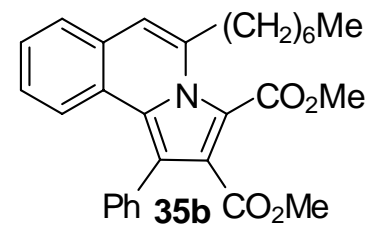

Pyrrolo-isoquinoline 35b: Yellow solid m.p. $63.0-64.0{ }^{\circ} \mathrm{C} ;{ }^{1} \mathrm{H}$ NMR $(400$ $\left.\mathrm{MHz}, \mathrm{CDCl}_{3}\right) \delta 7.48(\mathrm{~m}, 4 \mathrm{H}), 7.39(\mathrm{~m}, 2 \mathrm{H}), 7.30(\mathrm{~m}, 2 \mathrm{H}), 7.08(\mathrm{~m}, 1 \mathrm{H})$ $6.78(\mathrm{~s}, 1 \mathrm{H}), 4.00(\mathrm{~s}, 3 \mathrm{H}), 3.62(\mathrm{~s}, 3 \mathrm{H}), 2.93(\mathrm{t}, 2 \mathrm{H}, J=8.0 \mathrm{~Hz}), 1.78(\mathrm{~m}$, $2 \mathrm{H}), 1.37(\mathrm{~m}, 8 \mathrm{H}), 0.91(\mathrm{t}, 3 \mathrm{H}, J=6.9 \mathrm{~Hz}) ;{ }^{13} \mathrm{C} \mathrm{NMR}\left(100 \mathrm{MHz}, \mathrm{CDCl}_{3}\right) \delta$ $165.0,137.2,136.1,130.7,129.0,128.8,128.3,127.8,127.0,126.8,126.5$, 125.4, 123.5, 120.7, 120.3, 119.6, 113.2, 53.3, 51.8, 32.3, 32.0, 29.6, 29.3, 27.3, 22.9, 14.3; IR (thin film) vmax 3061, 3026, 2951, 2921, 2856, 1722, 1605, 1550, 1505, 1468, 1458, 1432, 1382, $1332,1251,1224,1208,1183,1162,1109,1095,945,762 \mathrm{~cm}^{-1}$; HRMS $(\mathrm{ESI}+)[\mathrm{M}+\mathrm{Na}]^{+}$ calculated for $\mathrm{C}_{29} \mathrm{H}_{31} \mathrm{NO}_{4} \mathrm{Na}: 480.2151$, found: 480.2156 .

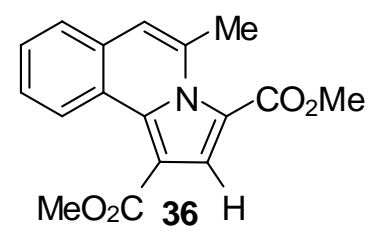

Pyrrolo-isoquinoline 36: White solid m.p. $126.0-128.0{ }^{\circ} \mathrm{C} ;{ }^{1} \mathrm{H}$ NMR $(400$ $\left.\mathrm{MHz}, \mathrm{CDCl}_{3}\right) \delta 9.73(\mathrm{~m}, 1 \mathrm{H}), 7.95(\mathrm{~s}, 1 \mathrm{H}), 7.59(\mathrm{~m}, 3 \mathrm{H}), 6.98(\mathrm{~s}, 1 \mathrm{H}), 3.94$ (s, 3H), $3.92(\mathrm{~s}, 3 \mathrm{H}), 2.69(\mathrm{~s}, 3 \mathrm{H}),{ }^{13} \mathrm{C}$ NMR $\left(100 \mathrm{MHz}, \mathrm{CDCl}_{3}\right) \delta 165.4$, $161.8,138.5,134.9,130.4,129.0,127.5,127.1,127.0,125.8,123.9,118.9$, 116.3, 109.2, 52.4, 51.9, 22.4; IR (thin film) vmax 3147, 3120, 3096, 3010, 2982, 2947, 2840, 1710, 1535, 1493, 1430, 1353, 1256, 1235, 1209, 1188, 1177, 1115, 1063, 1018, 957, 934, 836, $753 \mathrm{~cm}^{-1}$; HRMS (ESI+) $[\mathrm{M}+\mathrm{H}]^{+}$calculated for $\mathrm{C}_{17} \mathrm{H}_{16} \mathrm{NO}_{4}: 298.1079$, found: 298.1079. The structure of $\mathbf{3 6}$ was confirmed by x-ray crystal structure analysis (see page SI-16).

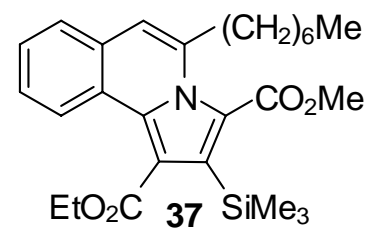

Pyrrolo-isoquinoline 37: Pale yellow solid m.p. 92.0-93.0 ${ }^{\circ} \mathrm{C} ;{ }^{1} \mathrm{H}$ NMR $\left(400 \mathrm{MHz}, \mathrm{CDCl}_{3}\right) \delta 8.35(\mathrm{~m}, 1 \mathrm{H}), 7.57(\mathrm{~m}, 1 \mathrm{H}), 7.44(\mathrm{~m}, 2 \mathrm{H}), 6.77(\mathrm{~s}, 1 \mathrm{H})$, $4.46(\mathrm{q}, 2 \mathrm{H}, J=7.2 \mathrm{~Hz}), 3.92(\mathrm{~s}, 3 \mathrm{H}), 2.86(\mathrm{t}, 2 \mathrm{H}, J=7.6 \mathrm{~Hz}), 1.70(\mathrm{~m}, 2 \mathrm{H})$, $1.44(\mathrm{t}, 3 \mathrm{H}, J=7.2 \mathrm{~Hz}), 1.32(\mathrm{~m}, 8 \mathrm{H}), 0.89(\mathrm{t}, 3 \mathrm{H}, J=6.8 \mathrm{~Hz}), 0.31(\mathrm{~s}, 9 \mathrm{H})$; ${ }^{13} \mathrm{C}$ NMR $\left(100 \mathrm{MHz}, \mathrm{CDCl}_{3}\right) \delta 169.3,165.4,137.2,131.5,128.7,127.1$, $126.8,126.1,124.38,124.36,123.7,123.5,115.5,112.5,61.3,52.2,32.5,31.6,29.2,28.9,27.2$, 22.5, 13.9, -0.001; IR (thin film) vmax 2983, 2954, 2926, 2853, 2367, 2359, 2341, 1720, 1473, $1458,1369,1350,1243,1188,1174,1114,1023,847,748 \mathrm{~cm}^{-1}$; HRMS $(\mathrm{ESI}+)[\mathrm{M}+\mathrm{Na}]^{+}$ calculated for $\mathrm{C}_{27} \mathrm{H}_{37} \mathrm{NO}_{4} \mathrm{SiNa}$ : 490.2390 , found: 490.2369 . The structure of 37 was determined by $\mathrm{x}$-ray crystal structure analysis (see page SI-18). 


\section{Mechanistic Studies}

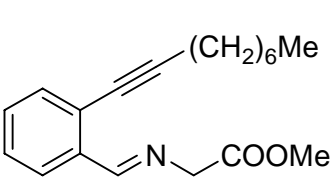

6

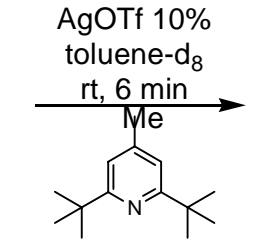

$\mathrm{MeO}_{2} \mathrm{C}=\mathrm{CO}_{2} \mathrm{Me}$<smiles>COC(=O)C1=C(C(=O)OC)N2C(C(C)C)=Cc3ccccc3C2C1C(C)C</smiles>

41

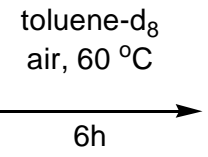<smiles>COC(=O)c1c(C(C)=O)c2c3ccccc3cc(C)n2c1C(C)C</smiles>

8

To an NMR tube was added alkynyl imine 6 (0.02 mmol, crude product from the corresponding alkynyl benzaldehyde), DMAD (4.9 $\mu \mathrm{L}, 0.04 \mathrm{mmol})$, DTBMP (8.2 $\mathrm{mg}, 0.04 \mathrm{mmol})$, and toluene$\mathrm{d}_{8}(1 \mathrm{~mL})$. The mixture was degassed at $-78{ }^{\circ} \mathrm{C}$ and the NMR tube charged with argon $(3 \mathrm{X})$. After acquisition of ${ }^{1} \mathrm{H}$ NMR data, $\operatorname{AgOTf}\left(50 \mu \mathrm{L}, 0.04 \mathrm{M}\right.$ in toluene- $\left.\mathrm{d}_{8}, 0.002 \mathrm{mmol}\right)$ was added and the reaction was monitored by ${ }^{1} \mathrm{H}$ NMR. Upon the consumption of alkynyl imine 6, the NMR tube cap was removed and the reaction was incubated at $60{ }^{\circ} \mathrm{C}$ for $6 \mathrm{~h}$ in the air to provide the final product.

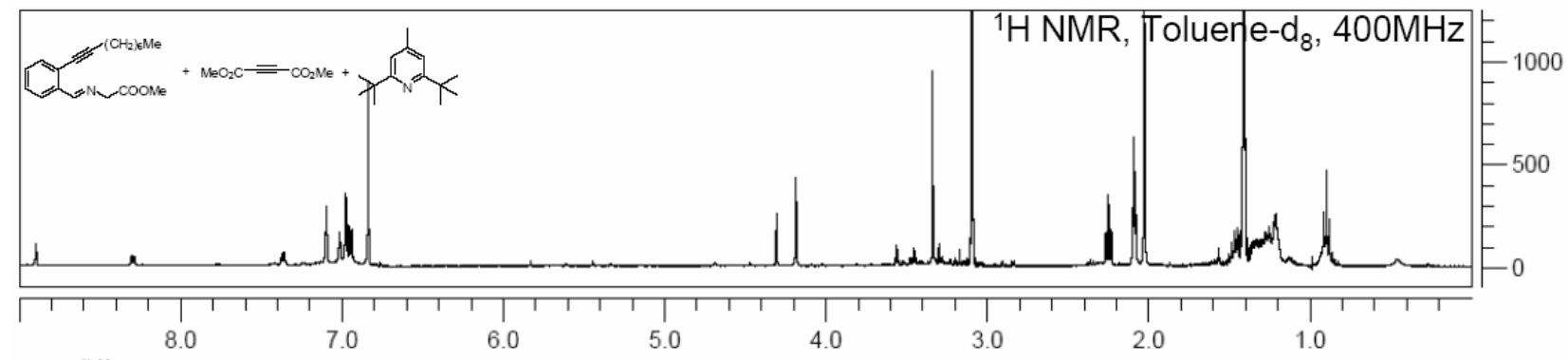

ppm (t1)

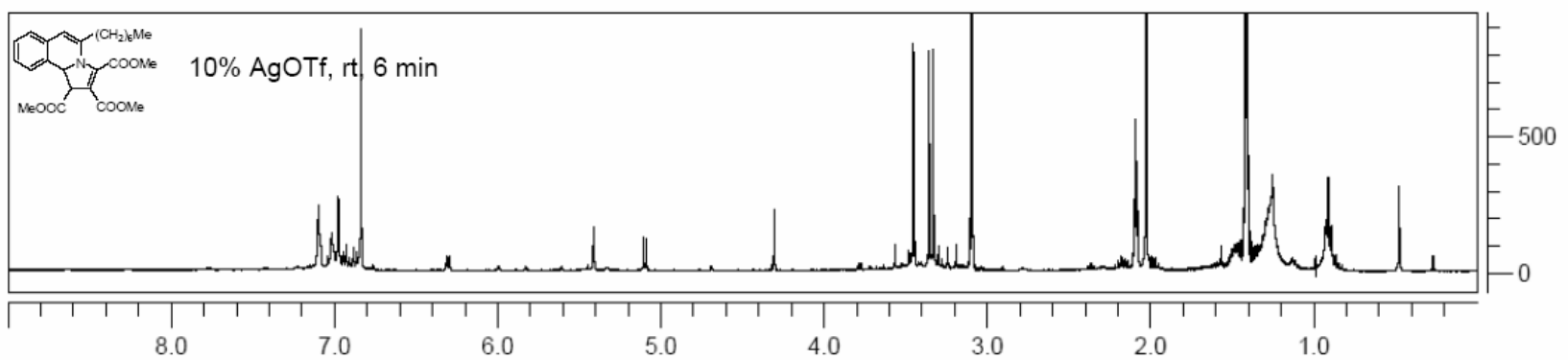

ppm (t1)

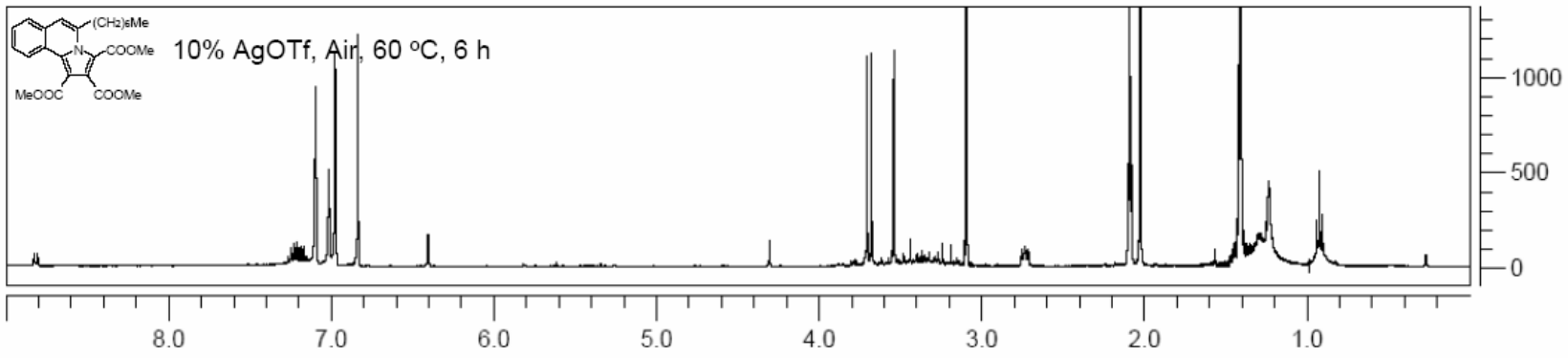

ppm (t1) 


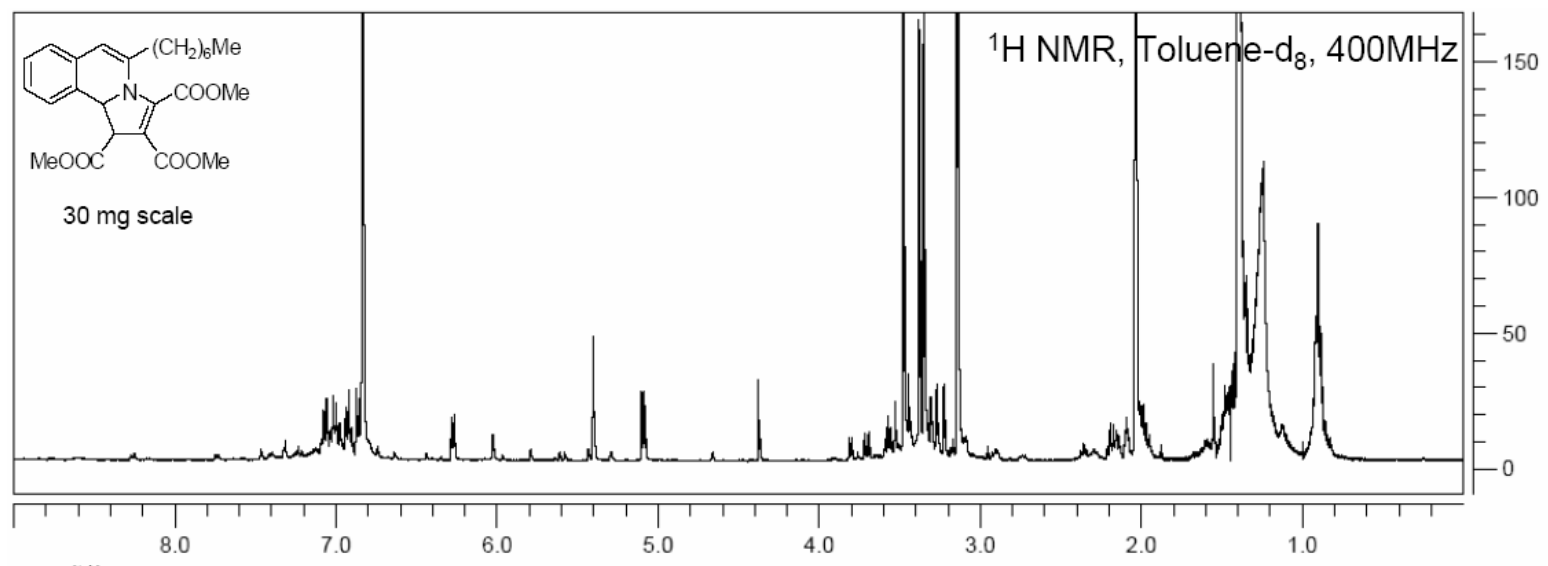

ppm (t1)
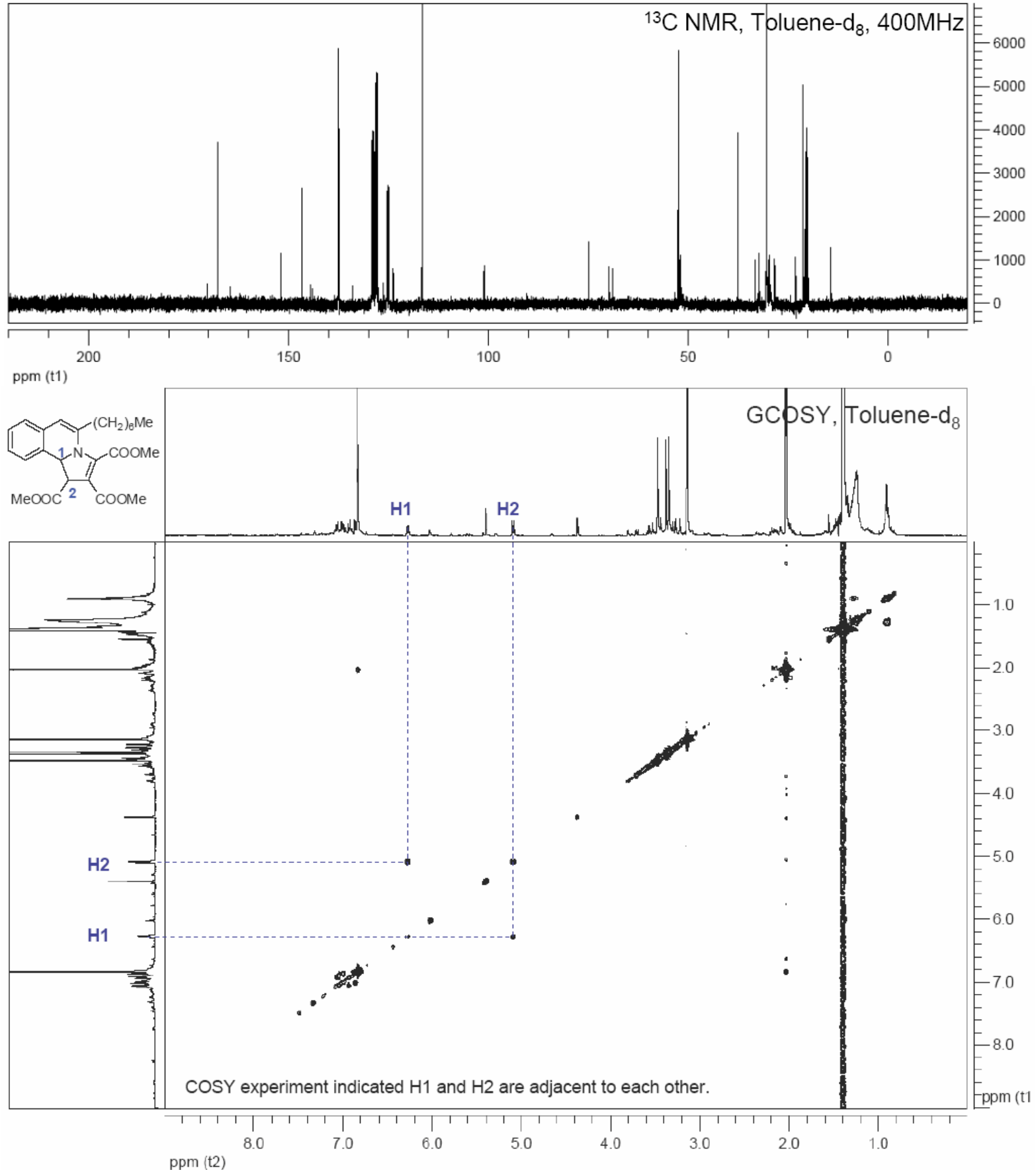

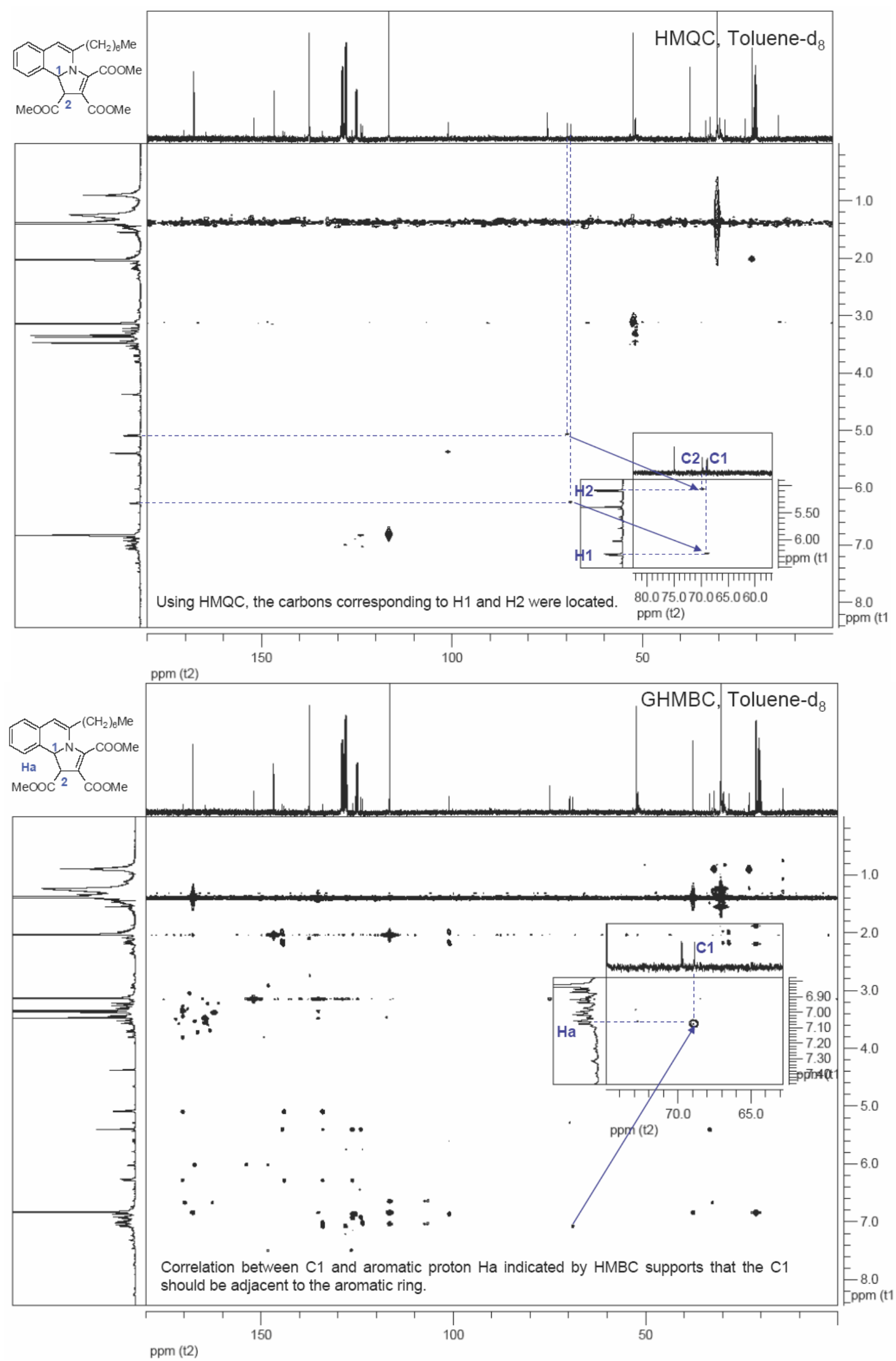

SI-11 


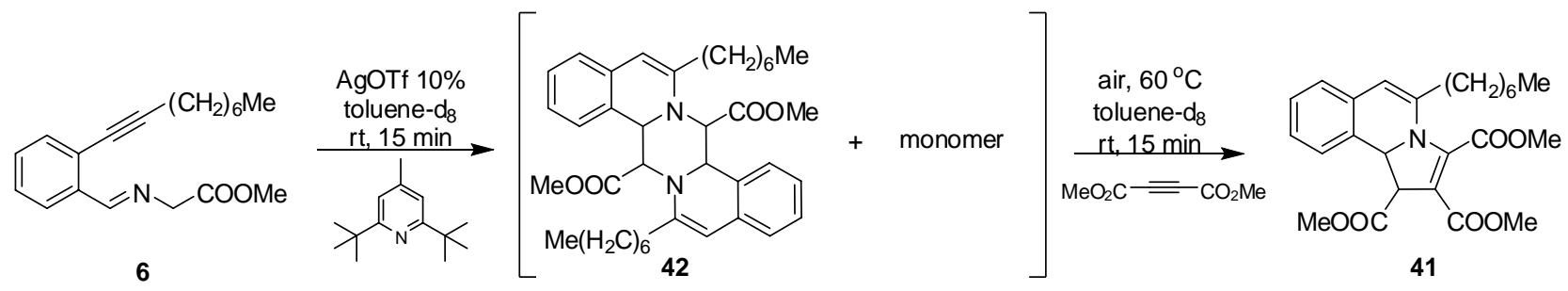

To a NMR tube was added alkynyl imine $6(0.02 \mathrm{mmol}$, crude product from the corresponding aldehyde), DTBMP $(8.2 \mathrm{mg}, 0.04 \mathrm{mmol})$, and toluene- $\mathrm{d}_{8}(1 \mathrm{~mL})$. The mixture was degassed at -78 ${ }^{\circ} \mathrm{C}$ and charged with argon (3X). After acquisition of ${ }^{1} \mathrm{H}$ NMR data, AgOTf $(50 \mu \mathrm{L}, 0.04 \mathrm{M}$ in toluene- $\mathrm{d}_{8}, 0.002 \mathrm{mmol}$ ) was added and the reaction was monitored by ${ }^{1} \mathrm{H}$ NMR. Upon the consumption of alkynyl imine, DMAD $(4.9 \mu \mathrm{L}, 0.04 \mathrm{mmol})$ was added at $\mathrm{rt}$ to provide 41.

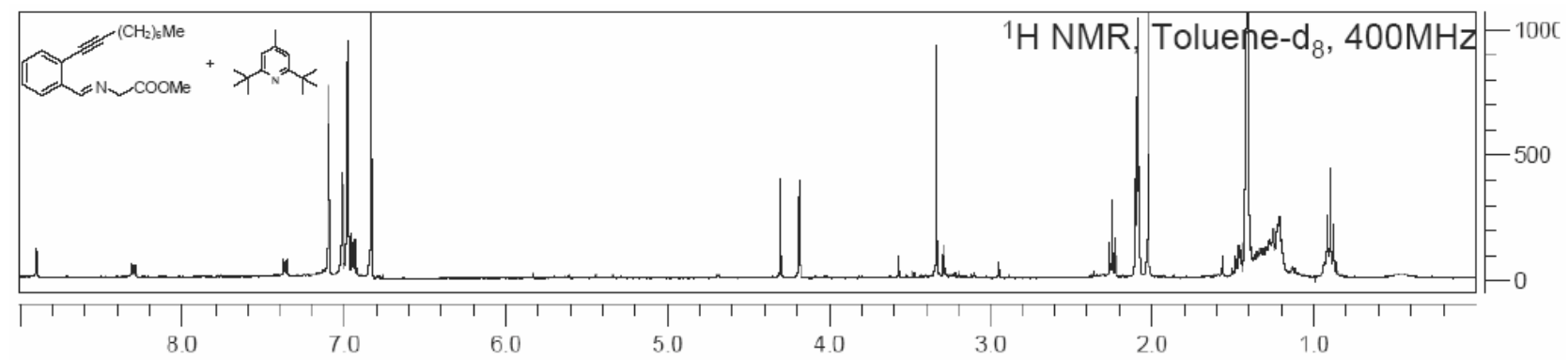

ppm (t1)

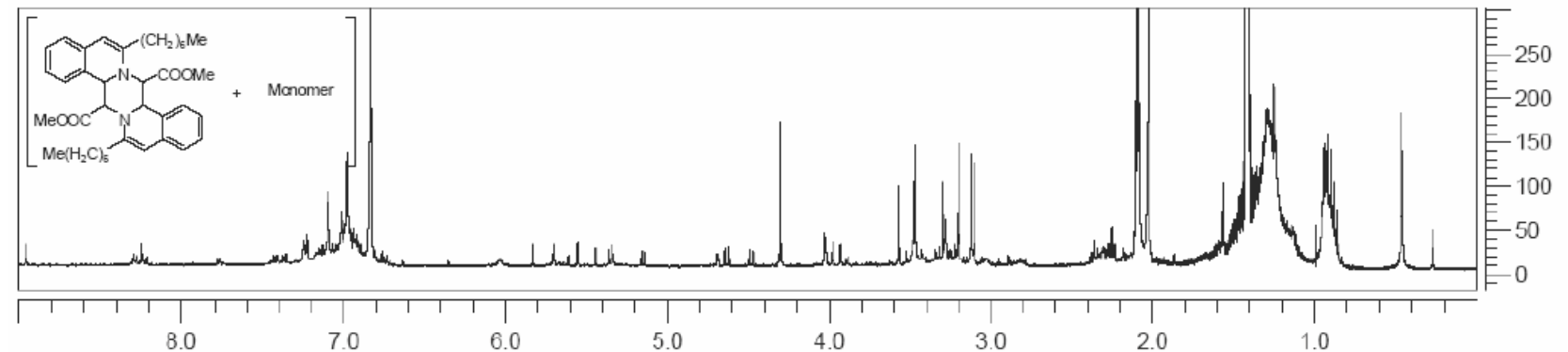

ppm (t1)

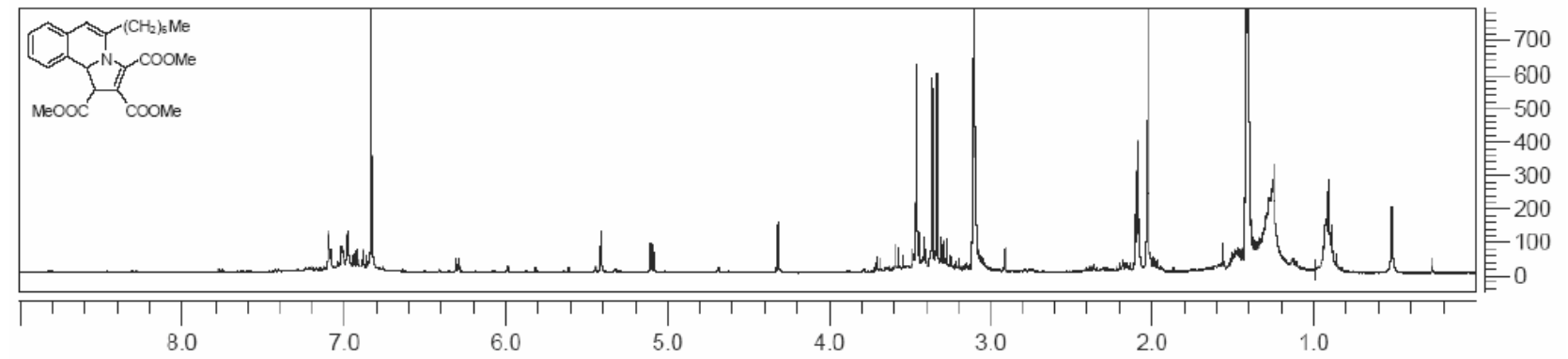

ppm (t1) 
To an NMR tube was added alkynyl imine 6 (0.02 mmol, crude product from the corresponding aldehyde), DTBMP (8.2 $\mathrm{mg}, 0.04 \mathrm{mmol})$, and toluene- $\mathrm{d}_{8}(1 \mathrm{~mL})$. The mixture was degassed at -78 ${ }^{\circ} \mathrm{C}$ and charged with argon (3X). After acquisition of ${ }^{1} \mathrm{H}$ NMR data, AgOTf $(50 \mu \mathrm{L}, 0.04 \mathrm{M}$ in toluene- $\mathrm{d}_{8}, 0.002 \mathrm{mmol}$ ) was added and the reaction was monitored by ${ }^{1} \mathrm{H}$ NMR. Upon the consumption of alkynyl imine, the reaction mixture was directly infused into a Micromass ZQ Quadrupole Mass Spectrometer (ESI+).

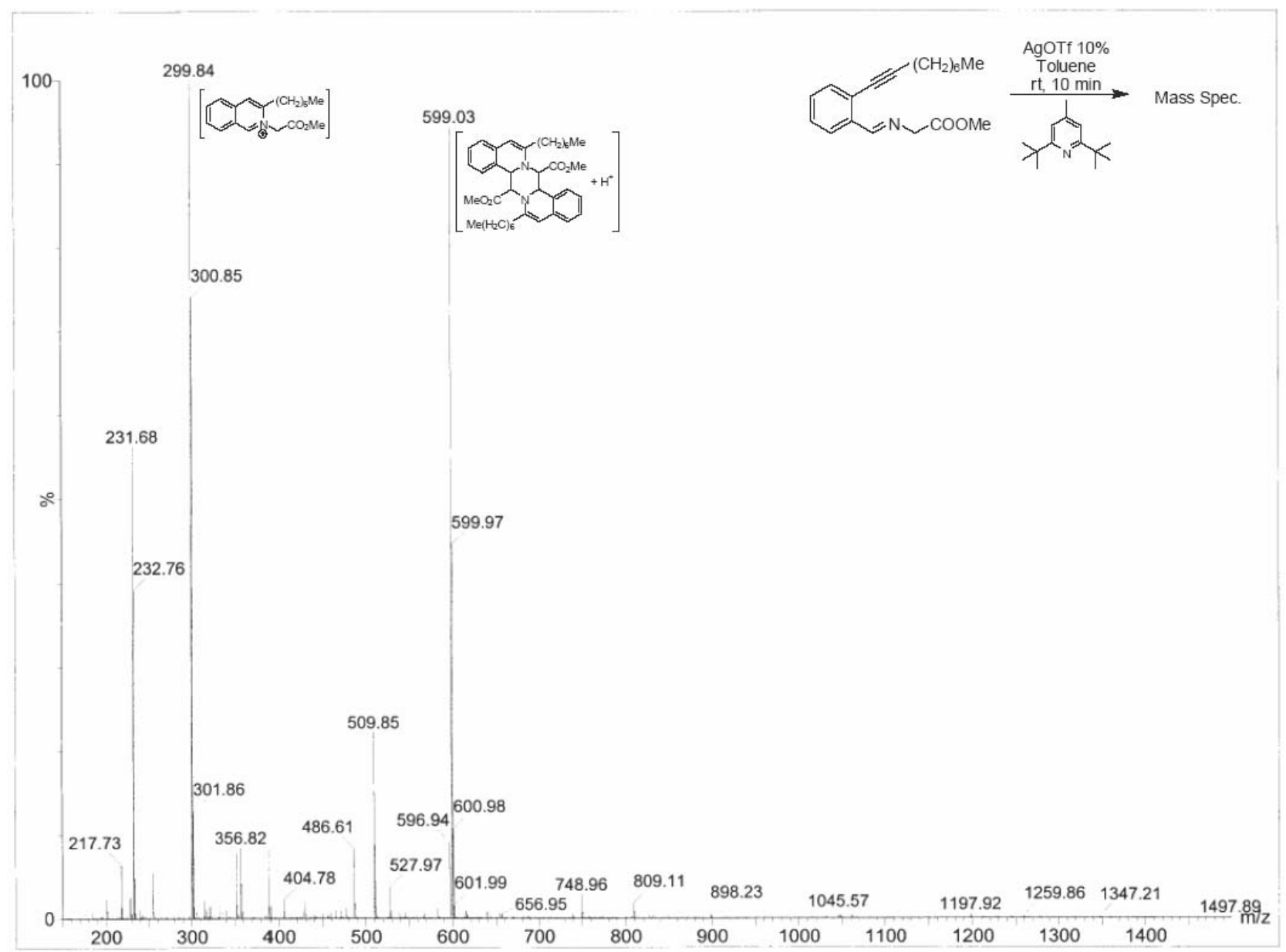




\section{X-ray Crystallographic Data}

Crystals of pyrrolo-isoquinoline 25 suitable for $\mathrm{x}$-ray analysis were obtained by slow evaporation from $\mathrm{CH}_{2} \mathrm{Cl}_{2} / 2,2$,4-trimethylpentane (1:1). Crystallographic data have been deposited with the Cambridge Crystallographic Data Centre (CCDC 644408). Copies of the data can be obtained free of charge on application to the CCDC, 12 Union Road, Cambridge CB21EZ, UK (fax: (+44)-1223-336-033; e-mail: deposit@ccdc.cam.ac.uk.)

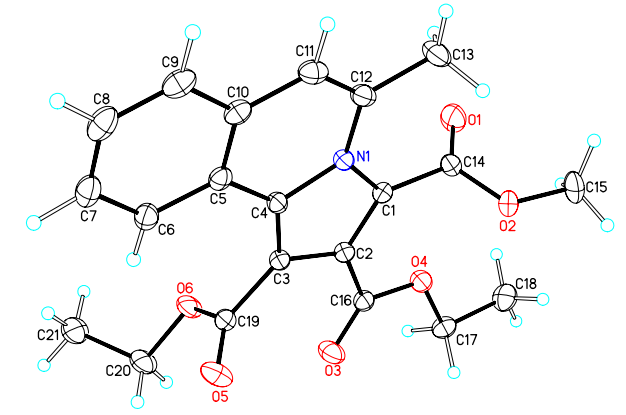

Table 1. Crystal data and structure refinement for 25.

Identification code

Empirical formula

Formula weight

Temperature

Wavelength

Crystal system

Space group

Unit cell dimensions

Volume

Z

Density (calculated)

Absorption coefficient

$\mathrm{F}(000)$

Crystal size

Theta range for data collection

Index ranges

Reflections collected

Independent reflections

Completeness to theta $=28.32^{\circ}$

Absorption correction

Max. and min. transmission
25

C21 H21 N O6

383.39

173(2) K

$0.71073 \AA$

Monoclinic

$\mathrm{P} 2(1) / \mathrm{n}$

$\mathrm{a}=10.6060(12) \AA$ $\alpha=90^{\circ}$.

$\mathrm{b}=7.1299(8) \AA$ $\beta=94.177(3)^{\circ}$.

$\mathrm{c}=24.583(3) \AA$ $\gamma=90^{\circ}$.
1854.0(4) $\AA^{3}$

4

$1.374 \mathrm{Mg} / \mathrm{m}^{3}$

$0.101 \mathrm{~mm}^{-1}$

808

$0.60 \times 0.50 \times 0.40 \mathrm{~mm}^{3}$

1.66 to $28.32^{\circ}$.

$-14<=\mathrm{h}<=14,-9<=\mathrm{k}<=9,-29<=1<=29$

16337

$4354[\mathrm{R}(\mathrm{int})=0.0316]$

$94.3 \%$

Semi-empirical from equivalents

0.9606 and 0.9418 
Refinement method

Data / restraints / parameters

Goodness-of-fit on $\mathrm{F}^{2}$

Final R indices [I $>2 \operatorname{sigma}(\mathrm{I})]$

$\mathrm{R}$ indices (all data)

Largest diff. peak and hole
Full-matrix least-squares on $\mathrm{F}^{2}$

4354 / 0 / 337

1.058

$\mathrm{R} 1=0.0381, \mathrm{wR} 2=0.1071$

$\mathrm{R} 1=0.0522, \mathrm{wR} 2=0.1138$

0.277 and -0.262 e. $\AA^{-3}$

Table 2. Atomic coordinates ( $\left.\mathrm{x} 10^{4}\right)$ and equivalent isotropic displacement parameters $\left(\AA^{2} \mathrm{x} 10^{3}\right)$ for 25. $U(e q)$ is defined as one third of the trace of the orthogonalized $U^{i j}$ tensor.

\begin{tabular}{|c|c|c|c|c|}
\hline & $\mathrm{x}$ & $\mathrm{y}$ & $\mathrm{z}$ & $\mathrm{U}(\mathrm{eq})$ \\
\hline $\mathrm{O}(1)$ & $677(1)$ & $9448(1)$ & $916(1)$ & $39(1)$ \\
\hline $\mathrm{O}(2)$ & $990(1)$ & $6562(1)$ & $1264(1)$ & $29(1)$ \\
\hline $\mathrm{O}(3)$ & $4340(1)$ & $8978(1)$ & $2359(1)$ & $33(1)$ \\
\hline $\mathrm{O}(4)$ & $2263(1)$ & $8955(1)$ & 2111(1) & $25(1)$ \\
\hline $\mathrm{O}(5)$ & $6786(1)$ & $7340(1)$ & 1681(1) & $35(1)$ \\
\hline $\mathrm{O}(6)$ & $6300(1)$ & 10411(1) & $1672(1)$ & $31(1)$ \\
\hline $\mathrm{N}(1)$ & $3371(1)$ & $7848(1)$ & $544(1)$ & $19(1)$ \\
\hline$C(1)$ & $2763(1)$ & $8152(1)$ & $1011(1)$ & $20(1)$ \\
\hline $\mathrm{C}(2)$ & $3667(1)$ & $8497(1)$ & $1431(1)$ & $21(1)$ \\
\hline$C(3)$ & $4867(1)$ & $8396(1)$ & $1218(1)$ & $19(1)$ \\
\hline$C(4)$ & $4686(1)$ & 7999(1) & $670(1)$ & $20(1)$ \\
\hline$C(5)$ & $5535(1)$ & $7763(1)$ & $244(1)$ & $21(1)$ \\
\hline$C(6)$ & $6852(1)$ & $8034(2)$ & $330(1)$ & $26(1)$ \\
\hline$C(7)$ & $7621(1)$ & $7767(2)$ & $-91(1)$ & $30(1)$ \\
\hline$C(8)$ & $7118(1)$ & $7229(2)$ & $-604(1)$ & $34(1)$ \\
\hline$C(9)$ & $5833(1)$ & $6977(2)$ & $-697(1)$ & $32(1)$ \\
\hline$C(10)$ & $5027(1)$ & $7244(2)$ & $-276(1)$ & $24(1)$ \\
\hline$C(11)$ & $3682(1)$ & $7021(2)$ & $-369(1)$ & $27(1)$ \\
\hline$C(12)$ & $2874(1)$ & $7320(2)$ & $17(1)$ & $24(1)$ \\
\hline$C(13)$ & $1472(1)$ & $7123(2)$ & $-88(1)$ & $33(1)$ \\
\hline$C(14)$ & $1362(1)$ & $8163(2)$ & $1049(1)$ & $23(1)$ \\
\hline$C(15)$ & $-330(1)$ & $6505(3)$ & $1382(1)$ & $45(1)$ \\
\hline$C(16)$ & $3485(1)$ & $8841(1)$ & $2008(1)$ & $21(1)$ \\
\hline$C(17)$ & $2007(1)$ & $9117(2)$ & $2684(1)$ & $26(1)$ \\
\hline$C(18)$ & $598(1)$ & $9166(2)$ & $2703(1)$ & $35(1)$ \\
\hline$C(19)$ & $6088(1)$ & $8618(2)$ & $1548(1)$ & $22(1)$ \\
\hline
\end{tabular}


Crystals of pyrrolo-isoquinoline 36 suitable for $\mathrm{x}$-ray analysis were obtained by slow evaporation from $\mathrm{CH}_{2} \mathrm{Cl}_{2} / 2$,2,4-trimethylpentane (1:1). Crystallographic data have been deposited with the Cambridge Crystallographic Data Centre (CCDC 644410). Copies of the data can be obtained free of charge on application to the CCDC, 12 Union Road, Cambridge CB21EZ, UK (fax: (+44)-1223-336-033; e-mail: deposit@ccdc.cam.ac.uk.)

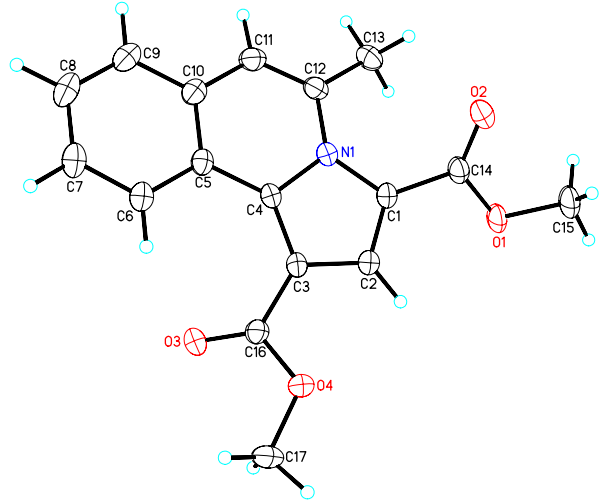

Table 3. Crystal data and structure refinement for 36.

Identification code

Empirical formula

Formula weight

Temperature

Wavelength

Crystal system

Space group

Unit cell dimensions

Volume

Z

Density (calculated)

Absorption coefficient

$\mathrm{F}(000)$

Crystal size

Theta range for data collection

Index ranges

Reflections collected

Independent reflections
36

C17 H15 N O4

297.30

173(2) K

$0.71073 \AA$

Triclinic

P-1

$\mathrm{a}=8.0265(8) \AA$ $\alpha=104.461(3)^{\circ}$.

$\mathrm{b}=9.2144(8) \AA$ $\beta=103.518(3)^{\circ}$.

$\mathrm{c}=10.8224(10) \AA$ $\gamma=108.685(3)^{\circ}$.

689.92(11) $\AA^{3}$

2

$1.431 \mathrm{Mg} / \mathrm{m}^{3}$

$0.103 \mathrm{~mm}^{-1}$

312

$0.60 \times 0.40 \times 0.10 \mathrm{~mm}^{3}$

2.07 to $27.52^{\circ}$.

$-10<=\mathrm{h}<=10,-11<=\mathrm{k}<=7,-13<=1<=14$

9161

$3100[\mathrm{R}(\mathrm{int})=0.0265]$ 
Completeness to theta $=27.52^{\circ}$

Absorption correction

Max. and min. transmission

Refinement method

Data / restraints / parameters

Goodness-of-fit on $\mathrm{F}^{2}$

Final $R$ indices [I $>2 \operatorname{sigma}(\mathrm{I})]$

$\mathrm{R}$ indices (all data)

Largest diff. peak and hole
$97.8 \%$

Semi-empirical from equivalents

0.9898 and 0.9409

Full-matrix least-squares on $\mathrm{F}^{2}$

$3100 / 0 / 259$

1.038

$\mathrm{R} 1=0.0396, \mathrm{wR} 2=0.0978$

$\mathrm{R} 1=0.0527, \mathrm{wR} 2=0.1039$

0.234 and -0.214 e. $\AA^{-3}$

Table 4. Atomic coordinates ( $\left.\times 10^{4}\right)$ and equivalent isotropic displacement parameters $\left(\AA^{2} \times 10^{3}\right)$ for 36. $U(\mathrm{eq})$ is defined as one third of the trace of the orthogonalized $\mathrm{U}^{\mathrm{ij}}$ tensor.

\begin{tabular}{|c|c|c|c|c|}
\hline & $\mathrm{x}$ & $\mathrm{y}$ & $\mathrm{z}$ & $\mathrm{U}(\mathrm{eq})$ \\
\hline $\mathrm{O}(1)$ & $2828(1)$ & $4729(1)$ & $-2890(1)$ & $30(1)$ \\
\hline $\mathrm{O}(2)$ & $-247(1)$ & $3195(1)$ & $-3936(1)$ & $34(1)$ \\
\hline $\mathrm{O}(3)$ & $2744(1)$ & $4637(1)$ & 2964(1) & $31(1)$ \\
\hline $\mathrm{O}(4)$ & $3495(1)$ & 6794(1) & $2277(1)$ & $28(1)$ \\
\hline $\mathrm{N}(1)$ & $-20(1)$ & $2246(1)$ & $-1430(1)$ & $20(1)$ \\
\hline $\mathrm{C}(1)$ & $1255(1)$ & $3524(1)$ & $-1640(1)$ & $22(1)$ \\
\hline $\mathrm{C}(2)$ & $2295(1)$ & $4733(1)$ & $-401(1)$ & $23(1)$ \\
\hline$C(3)$ & $1684(1)$ & $4250(1)$ & $599(1)$ & $21(1)$ \\
\hline$C(4)$ & $204(1)$ & 2703(1) & $-51(1)$ & 19(1) \\
\hline$C(5)$ & $-1069(1)$ & $1622(1)$ & $392(1)$ & $21(1)$ \\
\hline$C(6)$ & $-1208(1)$ & $2085(1)$ & $1696(1)$ & $26(1)$ \\
\hline$C(7)$ & $-2437(1)$ & $978(1)$ & 2051(1) & $31(1)$ \\
\hline$C(8)$ & $-3546(1)$ & $-619(1)$ & $1149(1)$ & $33(1)$ \\
\hline $\mathrm{C}(9)$ & $-3455(1)$ & $-1085(1)$ & $-129(1)$ & $29(1)$ \\
\hline$C(10)$ & $-2250(1)$ & $32(1)$ & $-539(1)$ & $23(1)$ \\
\hline $\mathrm{C}(11)$ & $-2157(1)$ & $-429(1)$ & $-1873(1)$ & $26(1)$ \\
\hline$C(12)$ & $-1089(1)$ & $623(1)$ & $-2321(1)$ & $24(1)$ \\
\hline$C(13)$ & $-867(2)$ & $82(1)$ & $-3670(1)$ & $32(1)$ \\
\hline$C(14)$ & $1145(1)$ & $3737(1)$ & $-2950(1)$ & $24(1)$ \\
\hline$C(15)$ & $2843(2)$ & $5174(1)$ & $-4081(1)$ & $36(1)$ \\
\hline$C(16)$ & $2636(1)$ & $5185(1)$ & $2057(1)$ & $23(1)$ \\
\hline$C(17)$ & $4574(2)$ & $7804(1)$ & $3672(1)$ & $36(1)$ \\
\hline
\end{tabular}


Crystals of pyrrolo-isoquinoline 37 suitable for $\mathrm{x}$-ray analysis were obtained by slow evaporation from $\mathrm{CH}_{2} \mathrm{Cl}_{2} / 2$,2,4-trimethylpentane (1:1). Crystallographic data have been deposited with the Cambridge Crystallographic Data Centre (CCDC 644409). Copies of the data can be obtained free of charge on application to the CCDC, 12 Union Road, Cambridge CB21EZ, UK (fax: (+44)-1223-336-033; e-mail: deposit@ccdc.cam.ac.uk.)

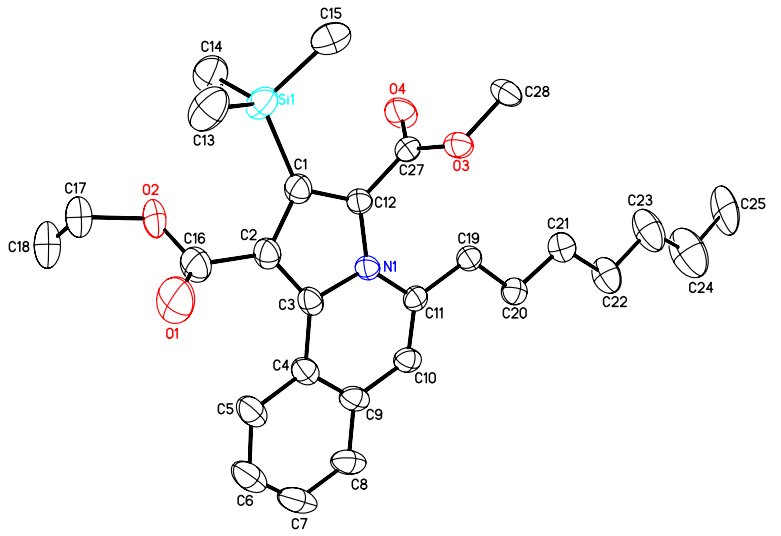

Table 5. Crystal data and structure refinement for 37.

Identification code

Empirical formula

Formula weight

Temperature

Wavelength

Crystal system

Space group

Unit cell dimensions

Volume

Z

Density (calculated)

Absorption coefficient

$\mathrm{F}(000)$

Crystal size

Theta range for data collection

Index ranges

Reflections collected

Independent reflections

Completeness to theta $=24.75^{\circ}$

Absorption correction

Max. and min. transmission
37

C27 H37 N O4 Si

467.67

223(2) K

$0.71073 \AA$

Monoclinic

$\mathrm{P} 2(1) / \mathrm{n}$

$\mathrm{a}=15.4051(16) \AA$

$\alpha=90^{\circ}$.

$\mathrm{b}=8.4192(8) \AA$

$\beta=97.664(5)^{\circ}$.

$\mathrm{c}=20.955(2) \AA$ $\gamma=90^{\circ}$.

2693.6(5) $\AA^{3}$

4

$1.153 \mathrm{Mg} / \mathrm{m}^{3}$

$0.118 \mathrm{~mm}^{-1}$

1008

$0.60 \times 0.20 \times 0.10 \mathrm{~mm}^{3}$

1.55 to $24.75^{\circ}$.

$-18<=\mathrm{h}<=18,-9<=\mathrm{k}<=9,-24<=\mathrm{l}<=24$

24115

$4596[\mathrm{R}(\mathrm{int})=0.0415]$

$99.5 \%$

Semi-empirical from equivalents

0.9883 and 0.9327 
Refinement method

Data / restraints / parameters

Goodness-of-fit on $\mathrm{F}^{2}$

Final R indices [I $>2 \operatorname{sigma}(\mathrm{I})]$

$\mathrm{R}$ indices (all data)

Largest diff. peak and hole
Full-matrix least-squares on $\mathrm{F}^{2}$

4596 / 22 / 336

1.059

$\mathrm{R} 1=0.0486, \mathrm{wR} 2=0.1301$

$\mathrm{R} 1=0.0787, \mathrm{wR} 2=0.1460$

0.719 and -0.280 e. $\AA^{-3}$

Table 6. Atomic coordinates $\left(\mathrm{x} 10^{4}\right)$ and equivalent isotropic displacement parameters $\left(\AA^{2} \mathrm{x} 10^{3}\right)$ for 37. $U(e q)$ is defined as one third of the trace of the orthogonalized $U^{i j}$ tensor.

\begin{tabular}{|c|c|c|c|c|}
\hline & $\mathrm{x}$ & $\mathrm{y}$ & $\mathrm{z}$ & $\mathrm{U}(\mathrm{eq})$ \\
\hline $\operatorname{Si}(1)$ & $4903(1)$ & $8481(1)$ & $2156(1)$ & $54(1)$ \\
\hline $\mathrm{O}(1)$ & $7186(1)$ & $9871(2)$ & $3427(1)$ & $99(1)$ \\
\hline $\mathrm{O}(2)$ & $5951(2)$ & $11270(3)$ & $2935(2)$ & $64(1)$ \\
\hline $\mathrm{O}\left(2^{\prime}\right)$ & $6095(2)$ & $11383(3)$ & $3155(2)$ & $57(1)$ \\
\hline $\mathrm{O}(3)$ & $3976(1)$ & $4860(1)$ & $3109(1)$ & $44(1)$ \\
\hline $\mathrm{O}(4)$ & $3095(1)$ & $6968(1)$ & $2925(1)$ & $60(1)$ \\
\hline$C(1)$ & $5056(1)$ & $8258(2)$ & $3062(1)$ & $44(1)$ \\
\hline $\mathrm{C}(2)$ & $5733(1)$ & $8933(2)$ & $3515(1)$ & $46(1)$ \\
\hline$C(3)$ & $5622(1)$ & $8453(2)$ & $4132(1)$ & $40(1)$ \\
\hline$C(4)$ & $6051(1)$ & $8840(2)$ & $4772(1)$ & $43(1)$ \\
\hline$C(5)$ & $6834(1)$ & $9716(2)$ & $4882(1)$ & $55(1)$ \\
\hline$C(6)$ & $7189(1)$ & $10103(2)$ & $5507(1)$ & $65(1)$ \\
\hline$C(7)$ & $6777(1)$ & $9653(2)$ & $6024(1)$ & $66(1)$ \\
\hline $\mathrm{C}(8)$ & $6020(1)$ & $8771(2)$ & $5928(1)$ & $54(1)$ \\
\hline $\mathrm{C}(9)$ & $5654(1)$ & $8332(2)$ & $5306(1)$ & $44(1)$ \\
\hline$C(10)$ & $4876(1)$ & $7381(2)$ & 5199(1) & $41(1)$ \\
\hline$C(11)$ & 4494(1) & $6937(2)$ & $4615(1)$ & $35(1)$ \\
\hline $\mathrm{N}(1)$ & $4877(1)$ & $7470(1)$ & $4074(1)$ & $35(1)$ \\
\hline$C(12)$ & $4558(1)$ & $7361(2)$ & $3423(1)$ & $37(1)$ \\
\hline$C(13)$ & $6000(1)$ & $8505(3)$ & $1867(1)$ & $77(1)$ \\
\hline$C(14)$ & $4277(1)$ & $10325(2)$ & $1928(1)$ & $69(1)$ \\
\hline$C(15)$ & $4291(2)$ & $6768(3)$ & $1750(1)$ & $77(1)$ \\
\hline$C(16)$ & $6408(1)$ & $10047(2)$ & $3337(1)$ & $60(1)$ \\
\hline$C(17)$ & $6508(3)$ & $12517(6)$ & $2663(2)$ & $91(2)$ \\
\hline $\mathrm{C}(18)$ & $6378(4)$ & $14032(7)$ & $3006(3)$ & $103(2)$ \\
\hline$C\left(17^{\prime}\right)$ & $6702(2)$ & $12536(5)$ & $2975(2)$ & $62(1)$ \\
\hline
\end{tabular}




\begin{tabular}{lrrrr}
$\mathrm{C}\left(18^{\prime}\right)$ & $6157(3)$ & $13973(5)$ & $2683(3)$ & $95(2)$ \\
$\mathrm{C}(19)$ & $3675(1)$ & $5957(2)$ & $4490(1)$ & $38(1)$ \\
$\mathrm{C}(20)$ & $3263(1)$ & $5468(2)$ & $5082(1)$ & $43(1)$ \\
$\mathrm{C}(21)$ & $2426(1)$ & $4527(2)$ & $4890(1)$ & $50(1)$ \\
$\mathrm{C}(22)$ & $1984(1)$ & $3938(2)$ & $5449(1)$ & $64(1)$ \\
$\mathrm{C}(23)$ & $1118(1)$ & $3083(3)$ & $5234(1)$ & $89(1)$ \\
$\mathrm{C}(24)$ & $631(2)$ & $2537(4)$ & $5737(2)$ & $124(1)$ \\
$\mathrm{C}(25)$ & $-262(4)$ & $1817(9)$ & $5548(4)$ & $100(3)$ \\
$\mathrm{C}\left(25^{\prime}\right)$ & $-311(3)$ & $2061(7)$ & $5409(4)$ & $178(3)$ \\
$\mathrm{C}(27)$ & $3790(1)$ & $6403(2)$ & $3140(1)$ & $39(1)$ \\
$\mathrm{C}(28)$ & $3262(1)$ & $3868(2)$ & $2807(1)$ & $60(1)$ \\
\hline
\end{tabular}




\section{Select NMR Spectra}

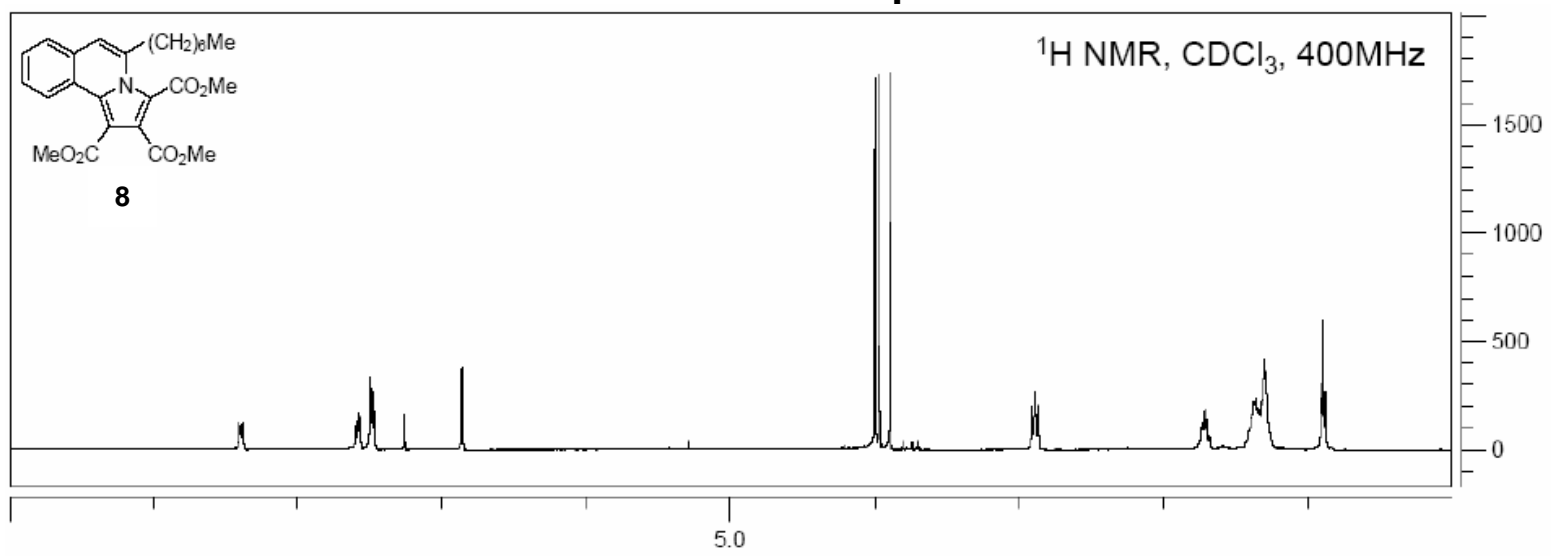

ppm (t1)
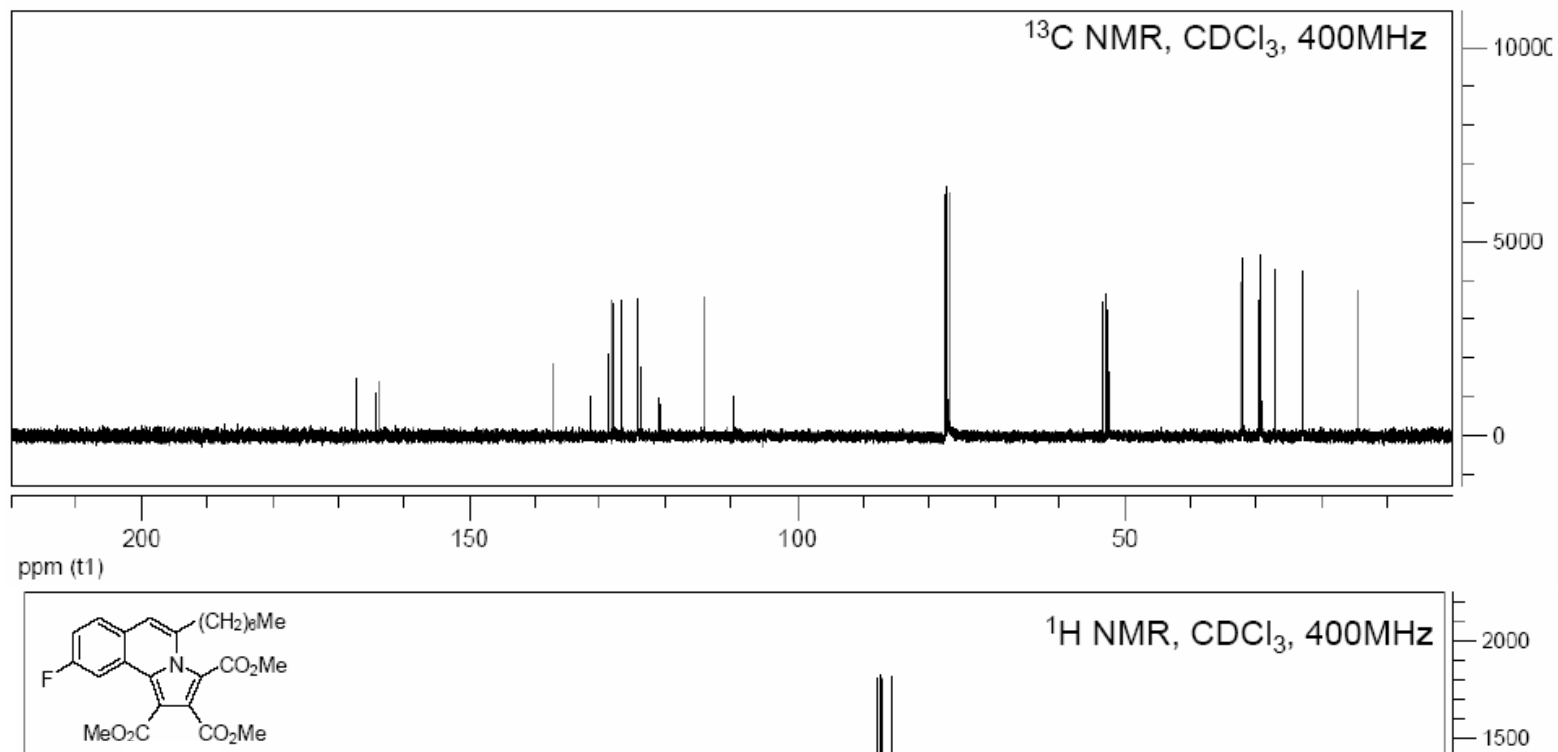

24

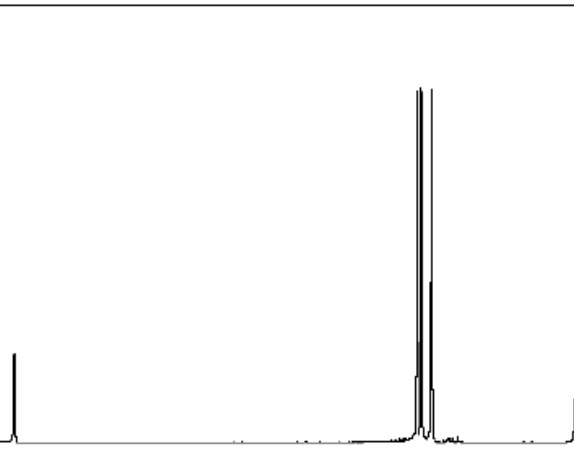

${ }^{1} \mathrm{H} \mathrm{NMR}, \mathrm{CDCl}_{3}, 400 \mathrm{MHz} \mathbb{E}_{2000}$

a.

5.0

ppm (t1)

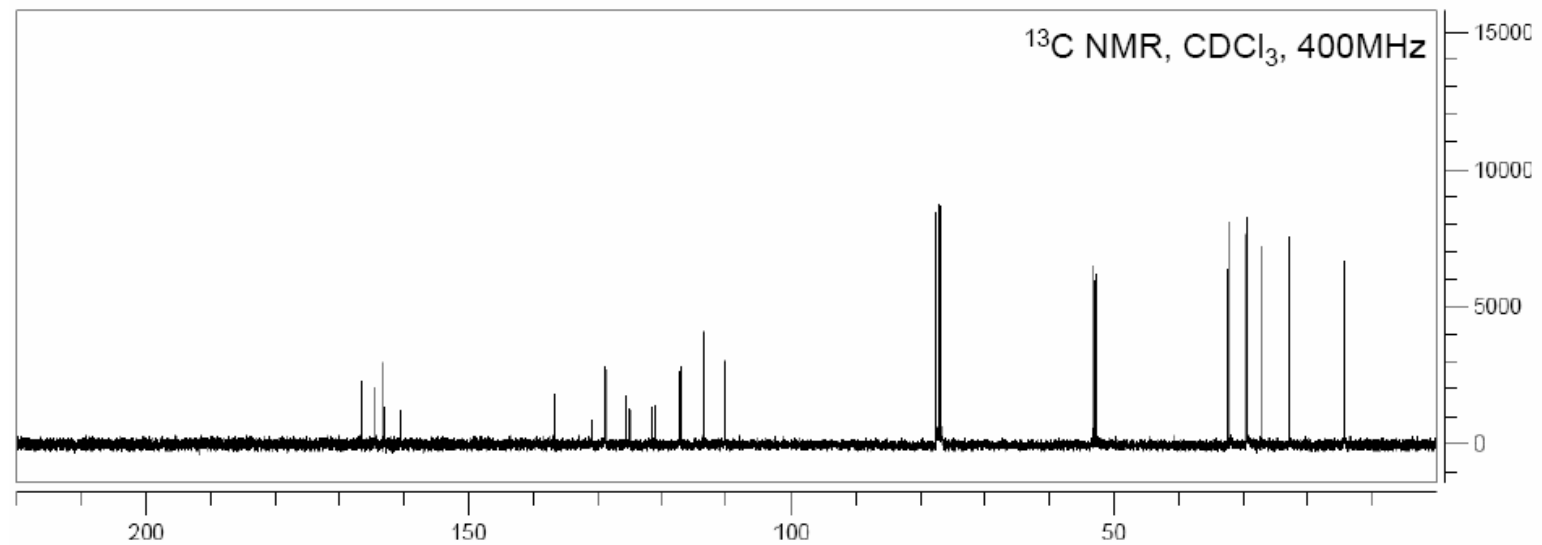

ppm (t1) 


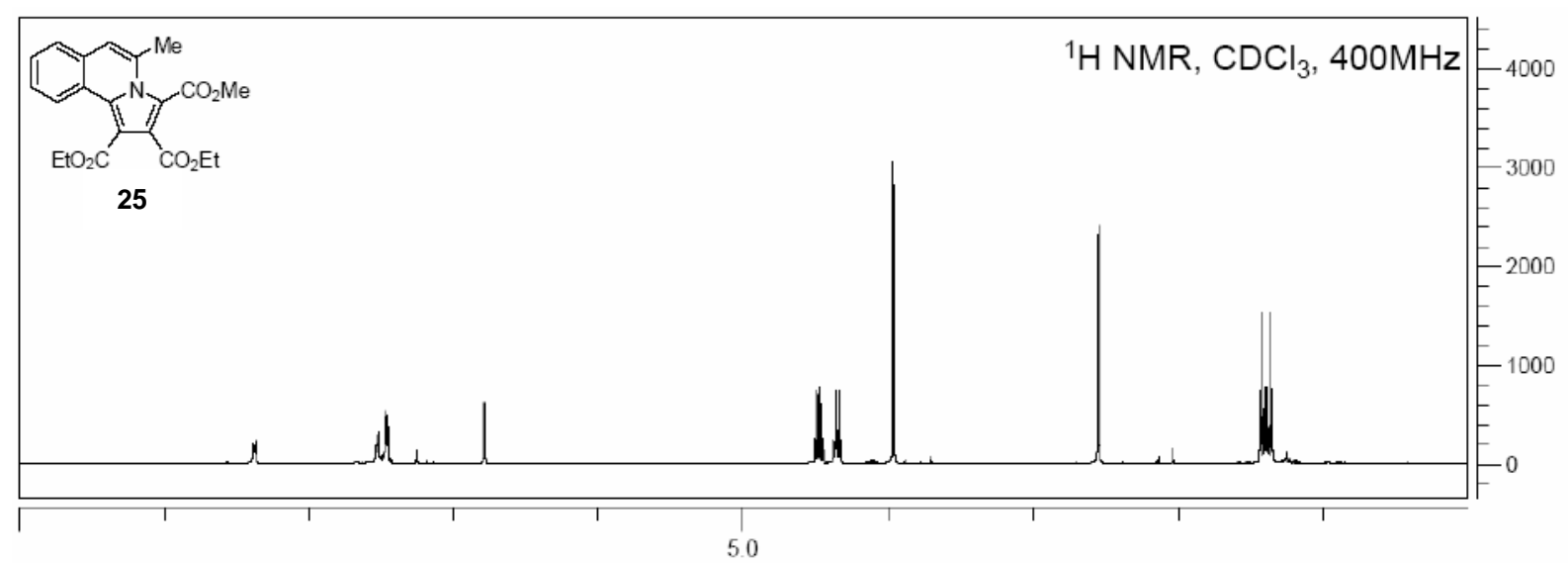

ppm (t1)

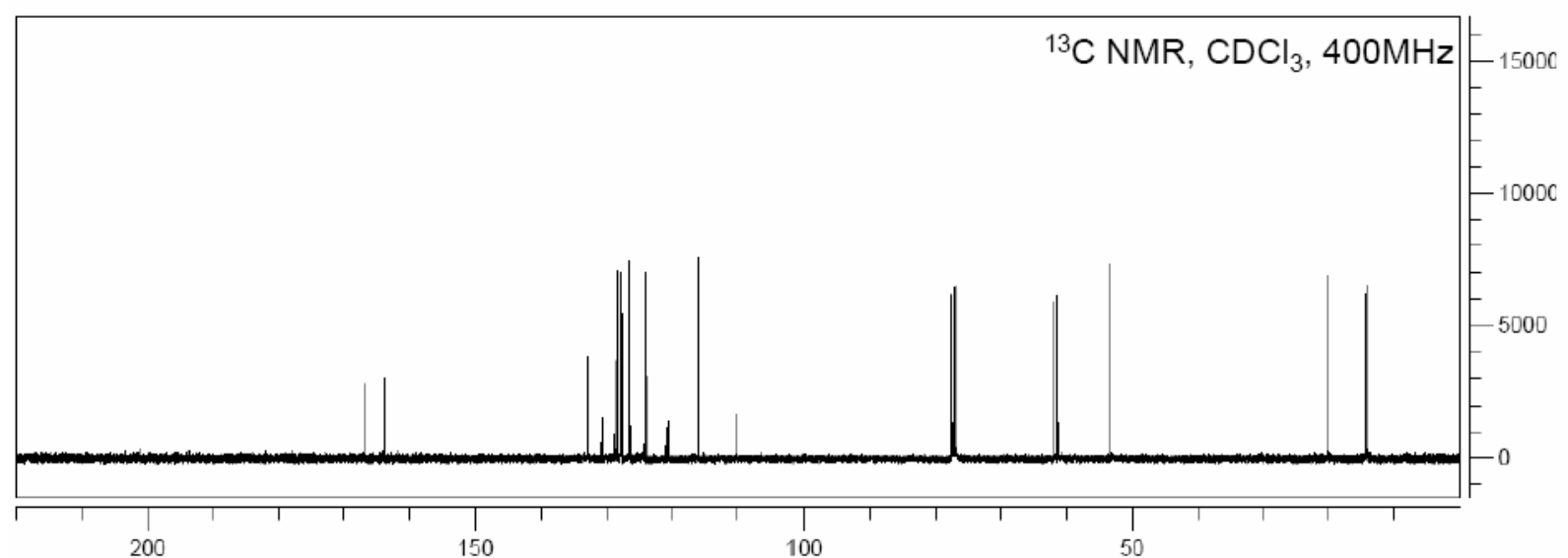

(t1)

26

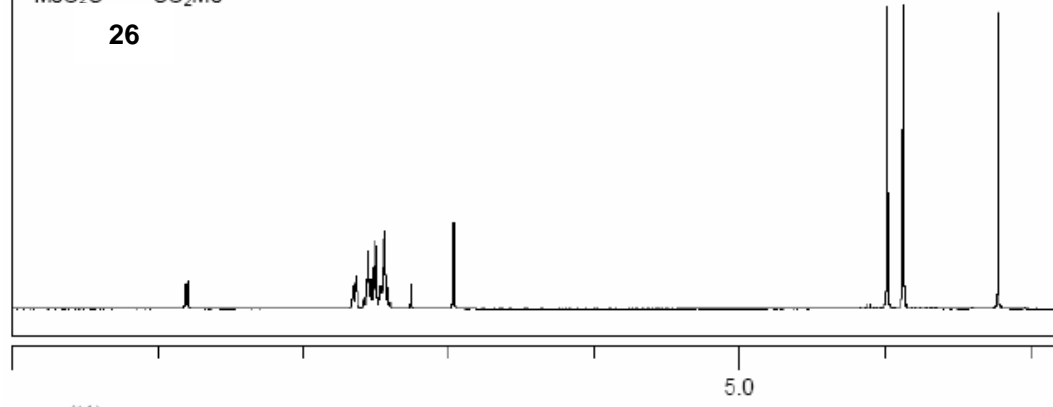

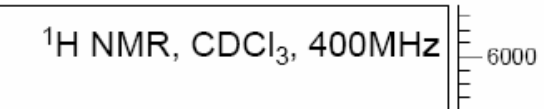
5000 4000 3000 2000 $=1000$ ppm (t1)

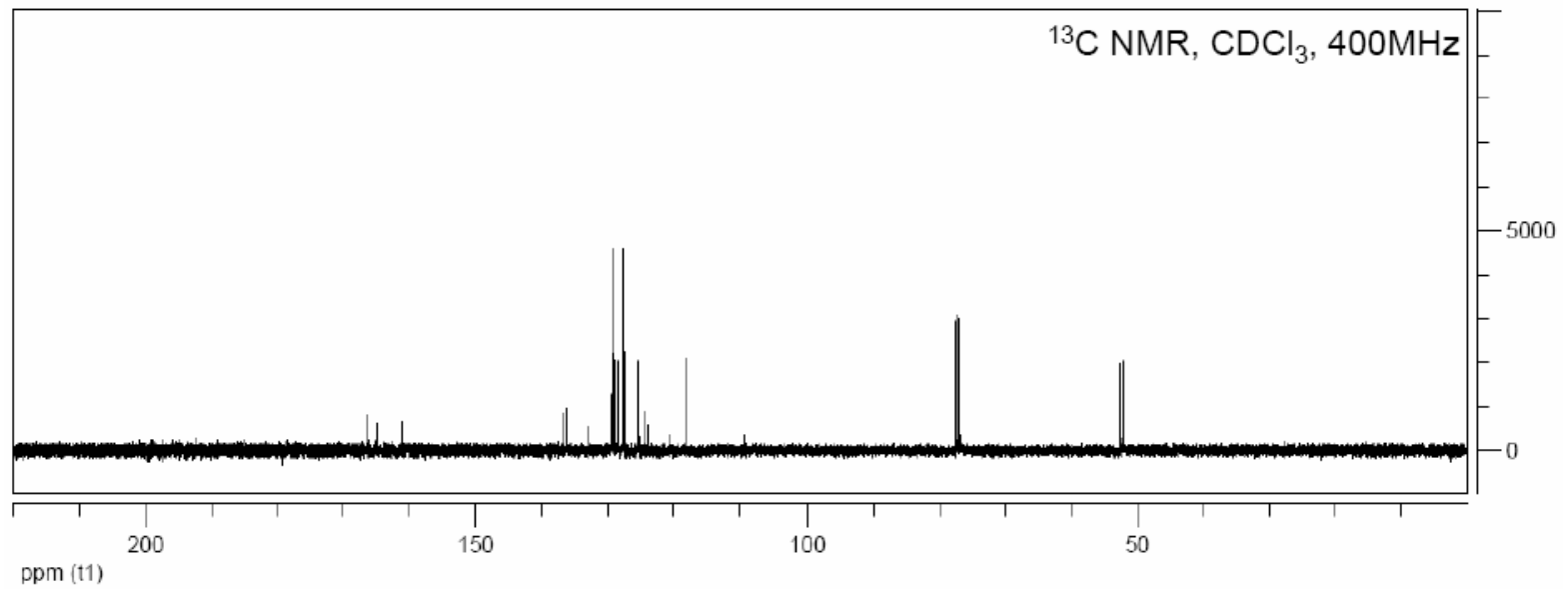




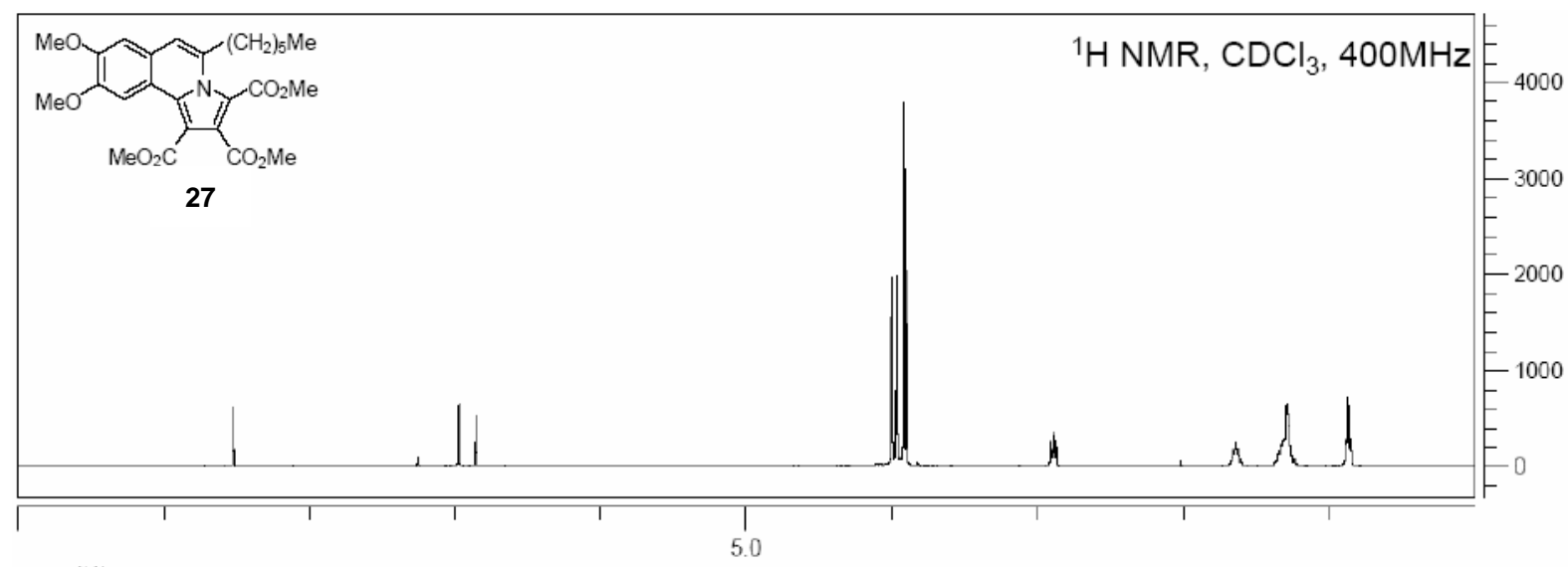

ppm (t1)
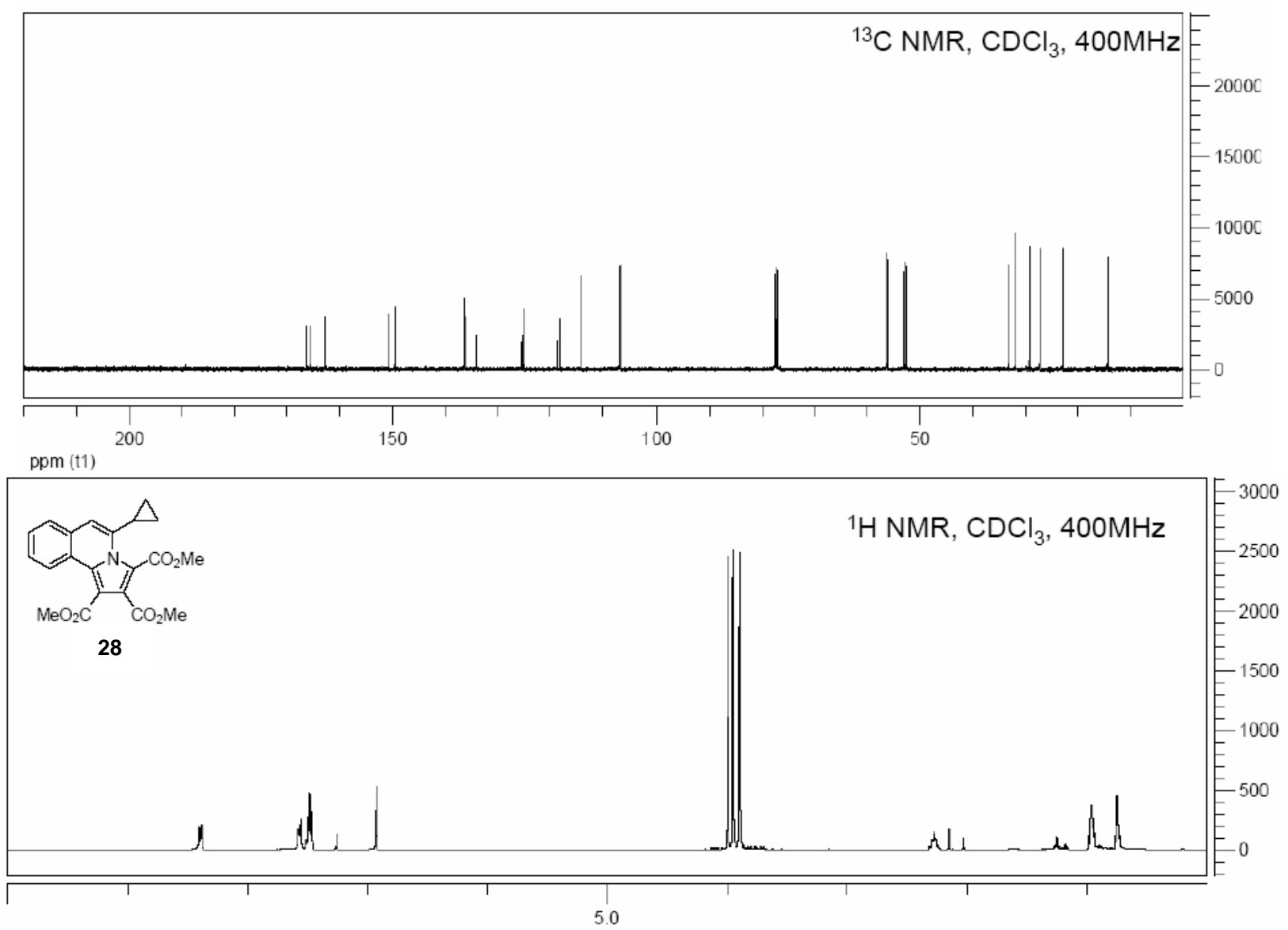

ppm (t1)

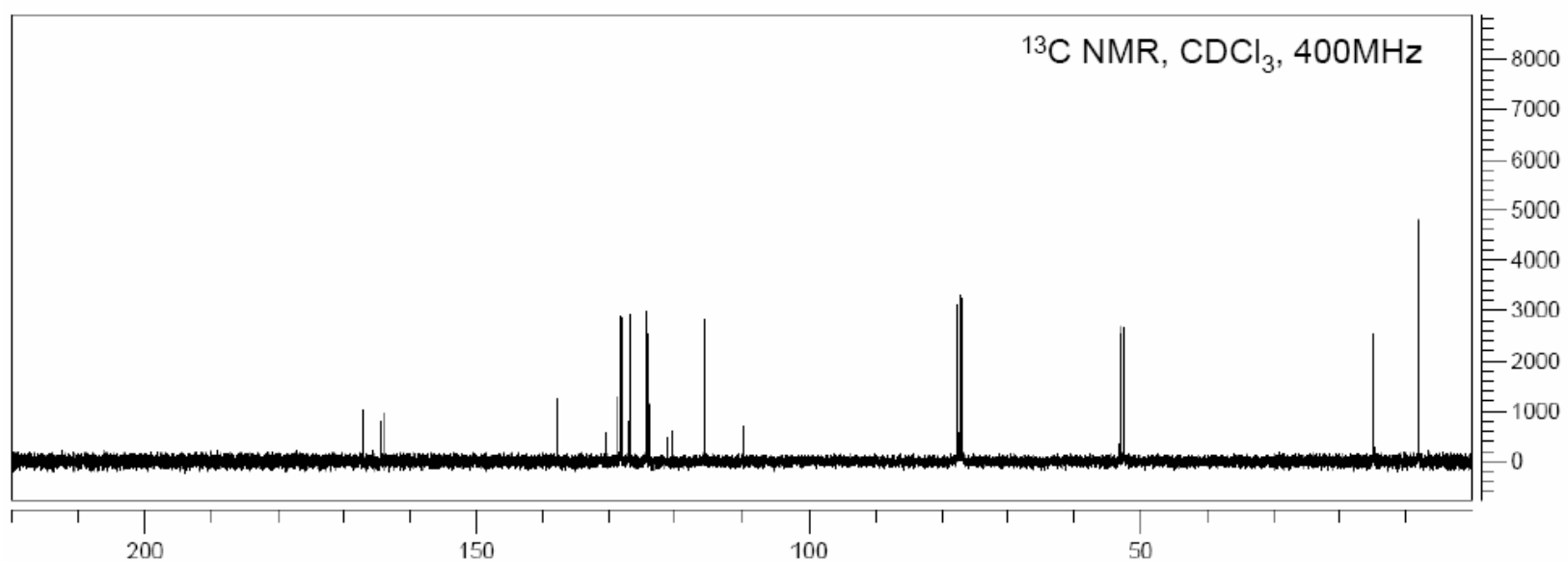

SI-23 


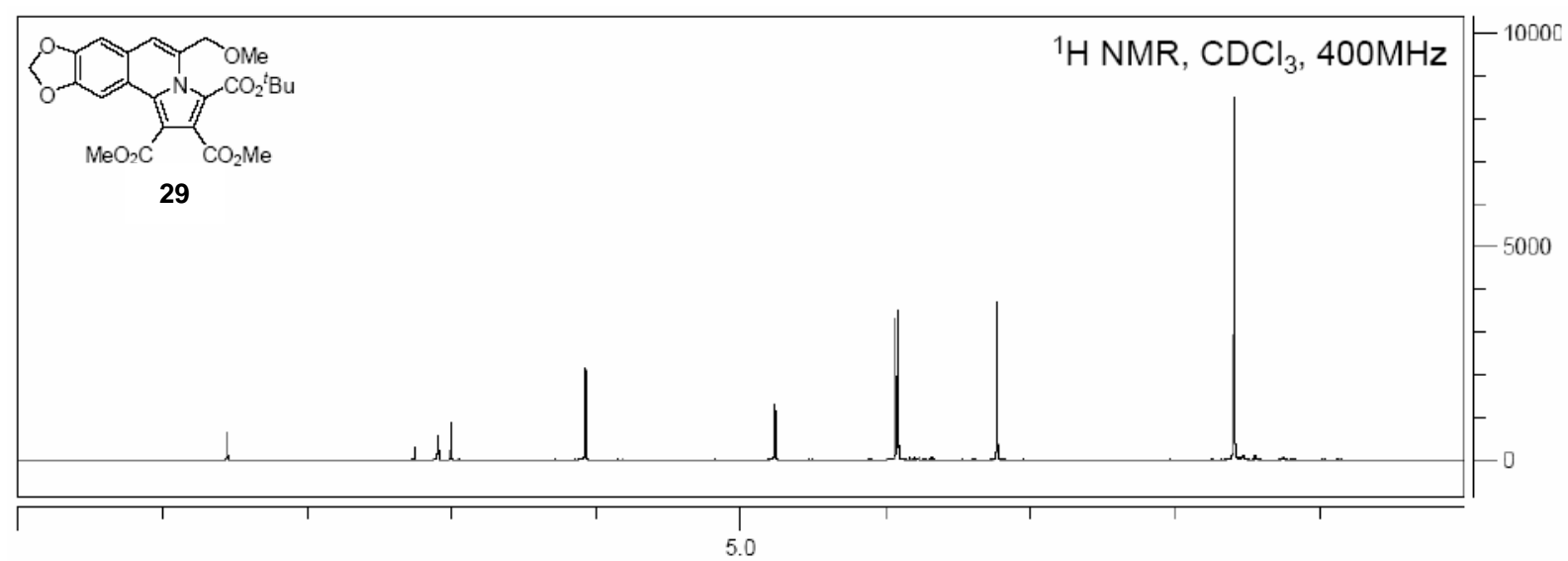

ppm (t1)

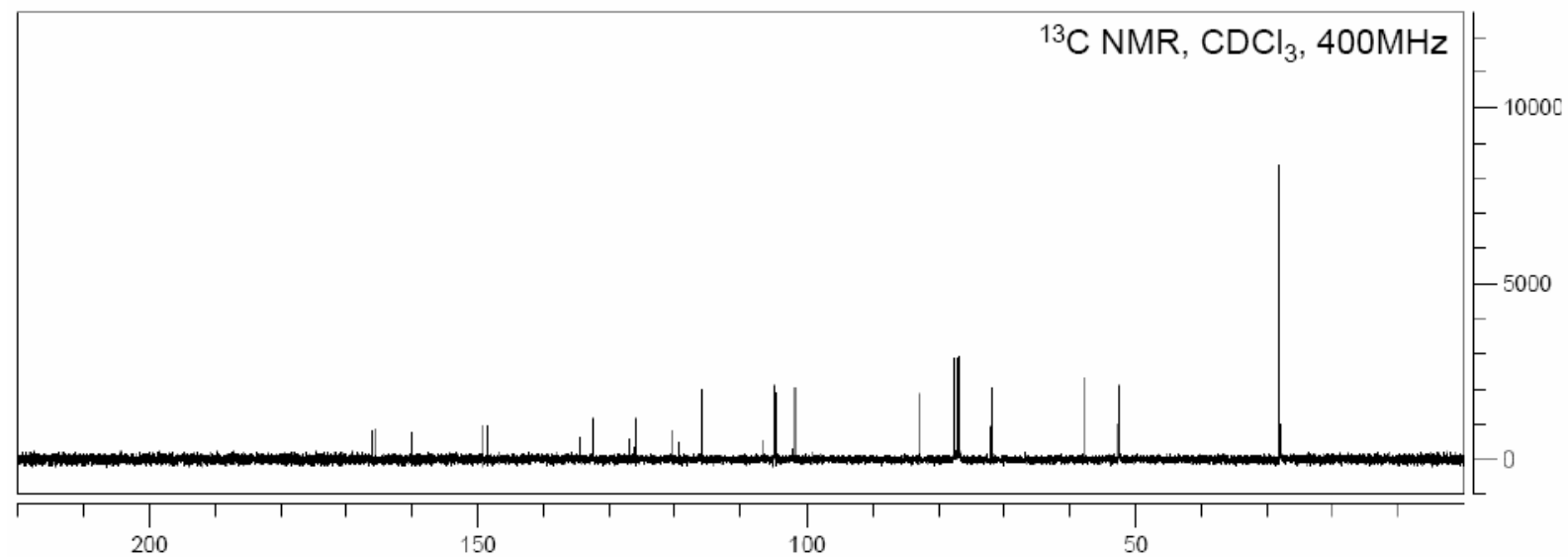

ppm (t1)

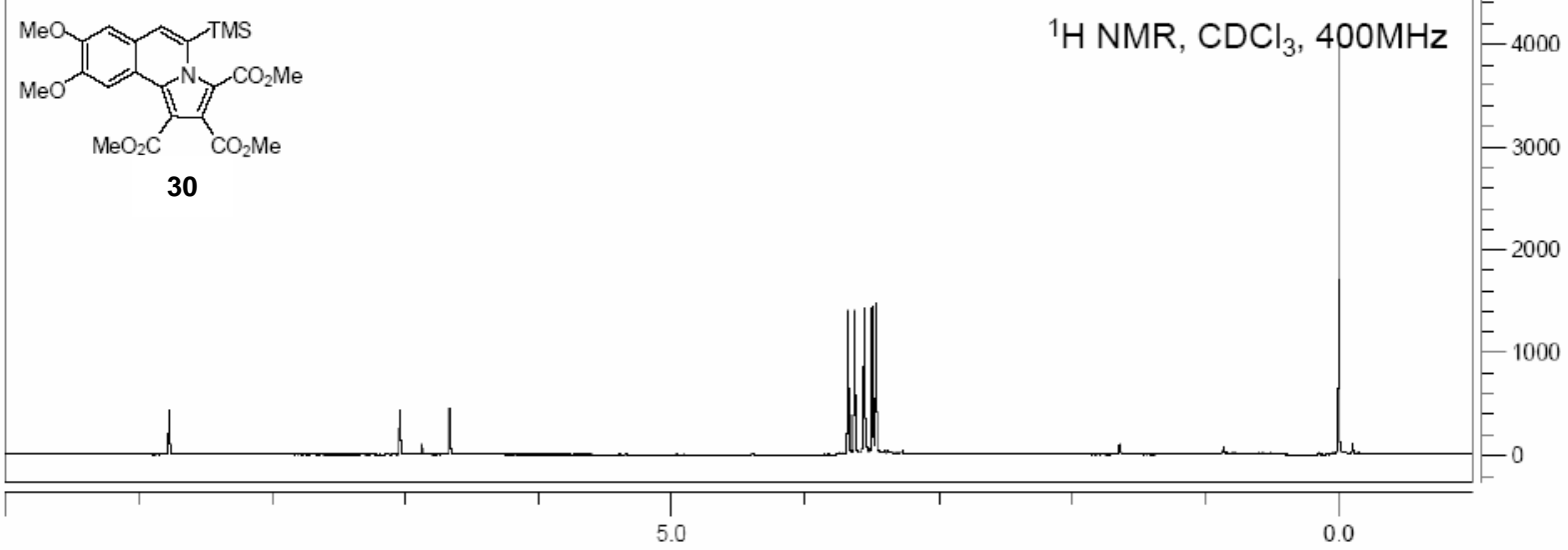

ppm (t1)

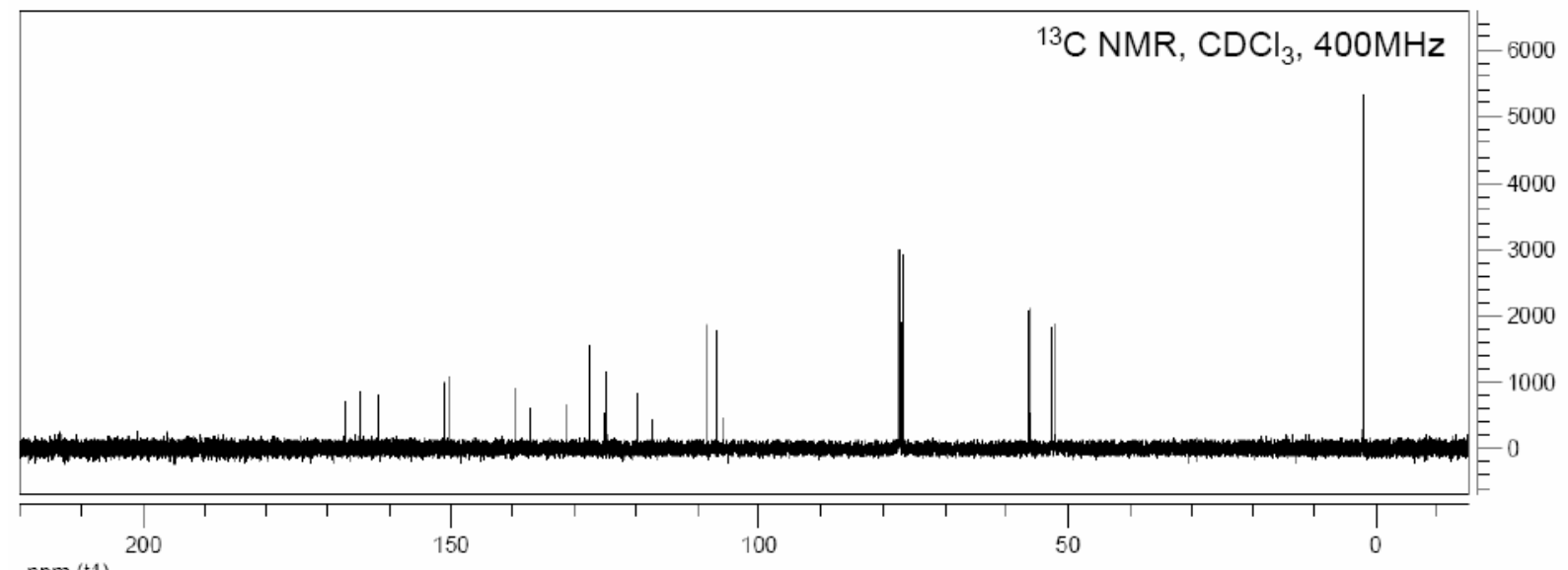
ppm (t1) 


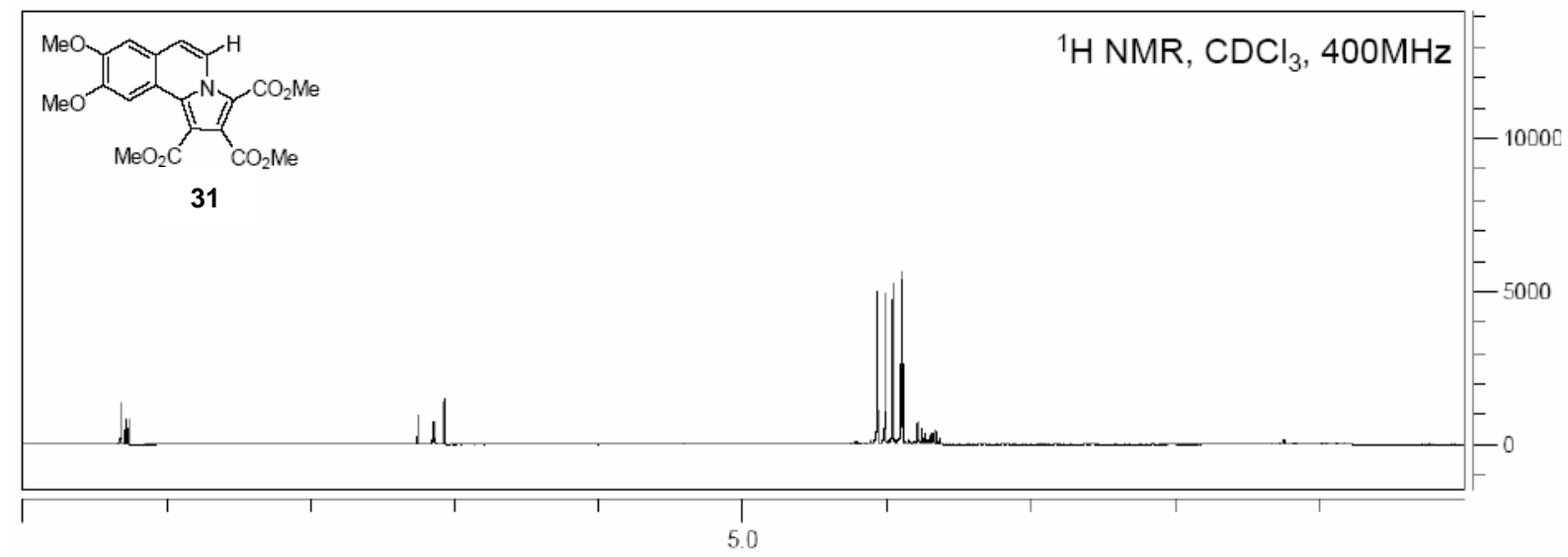

ppm (t1)
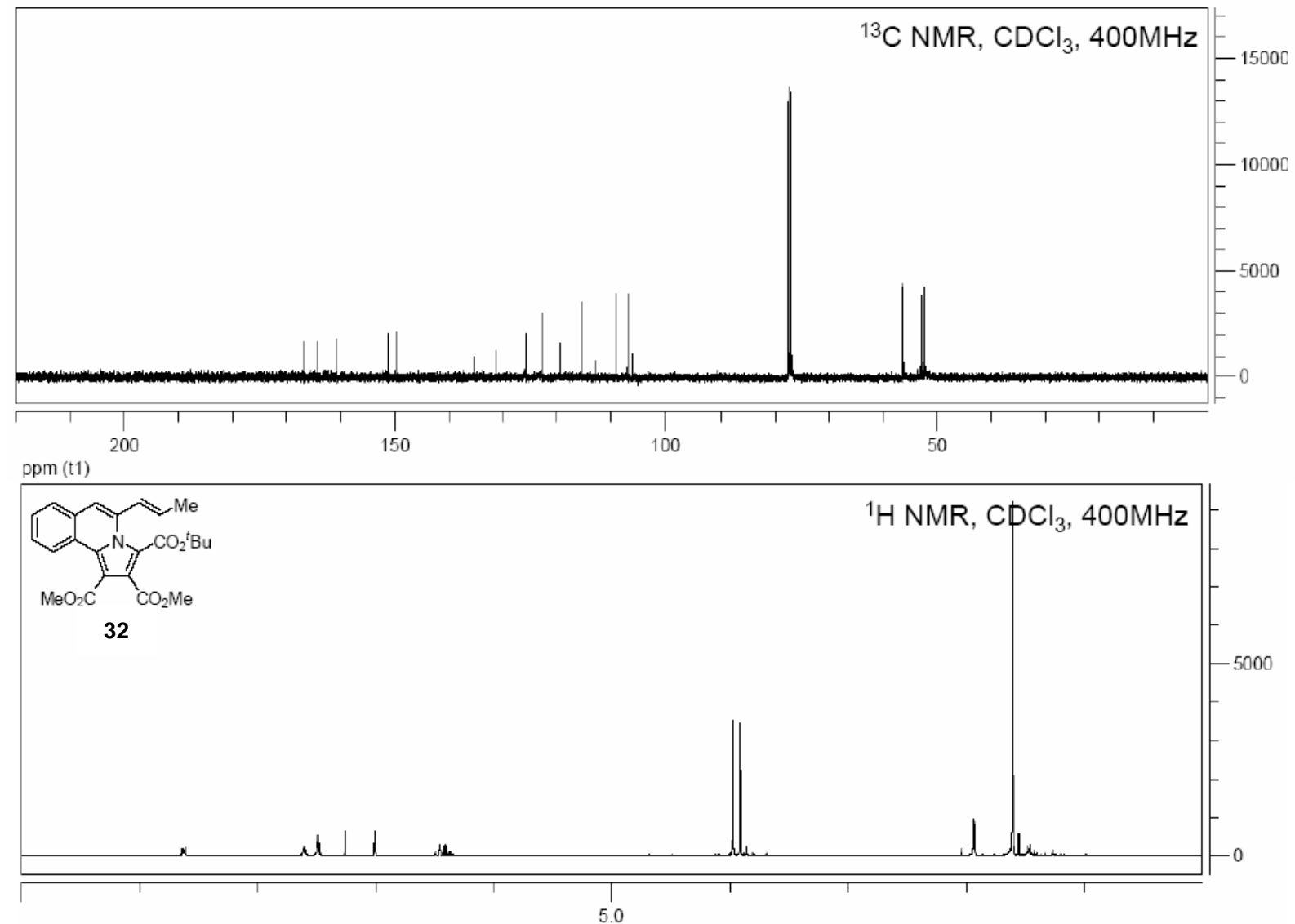

ppm (t1)

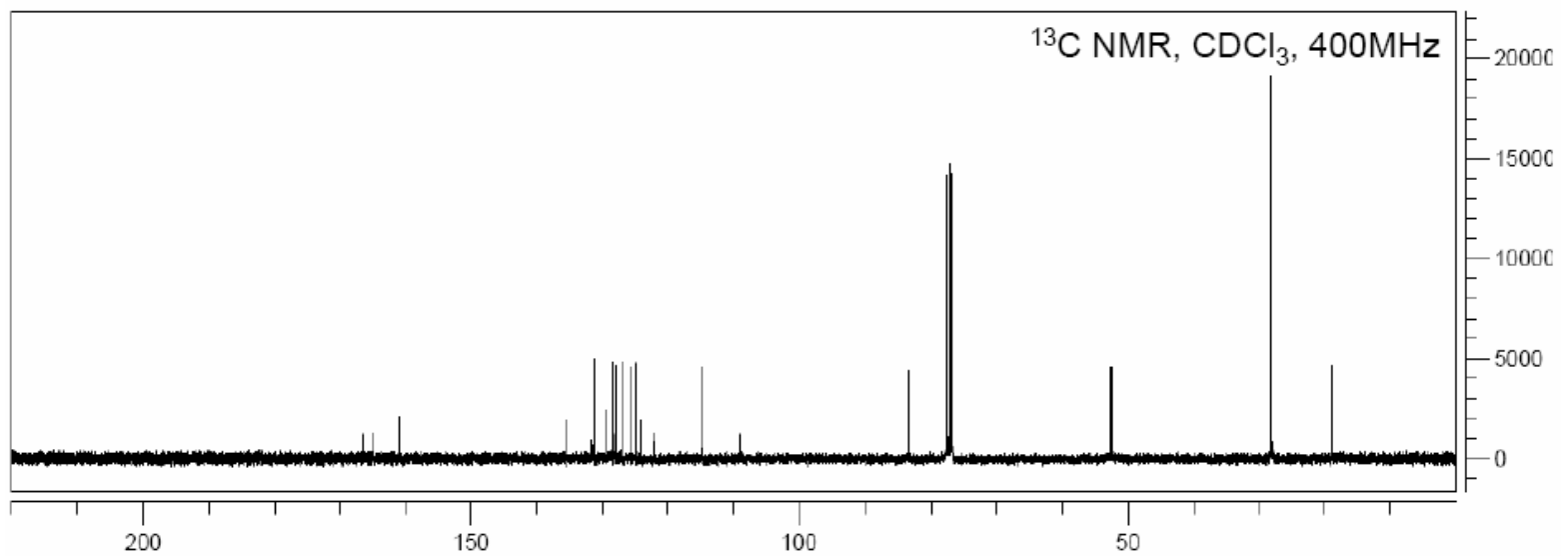

ppm (t1) 


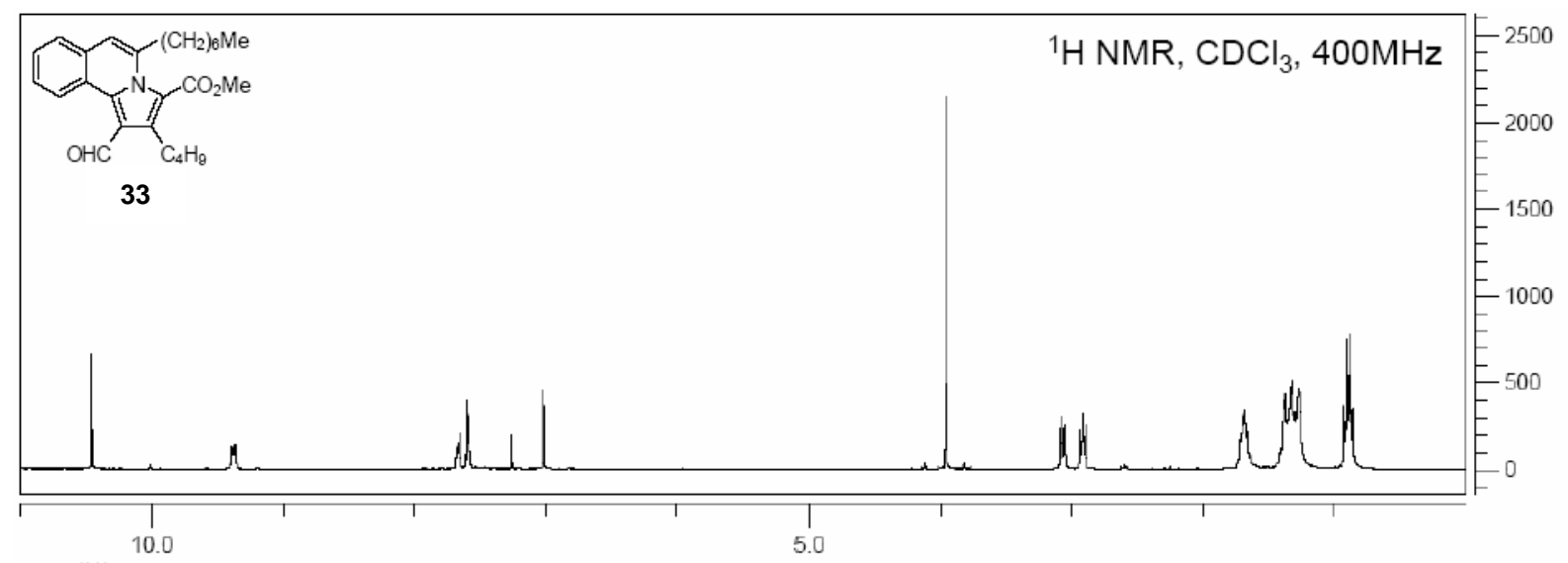

ppm (t1)
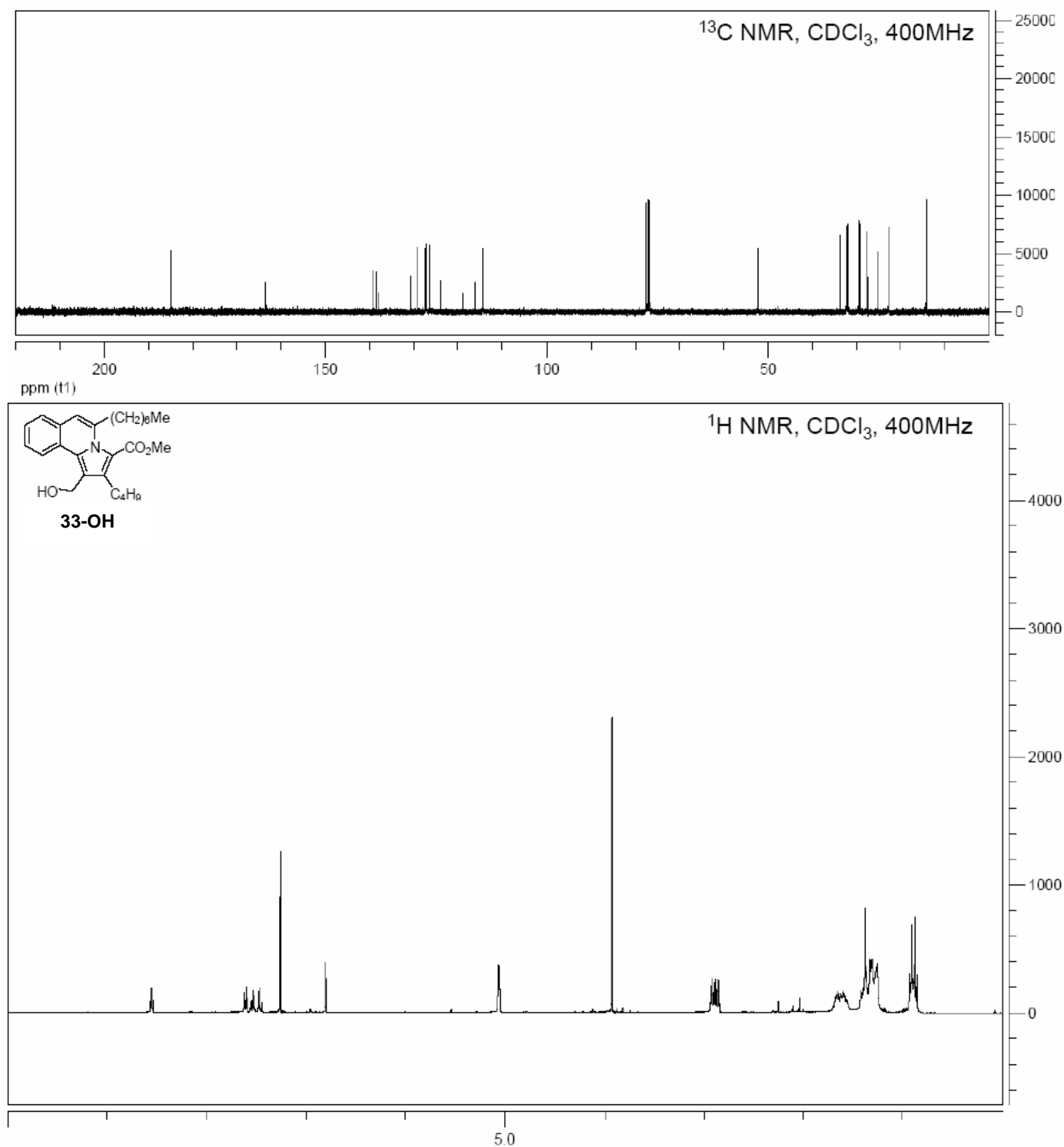

ppm (t1) 

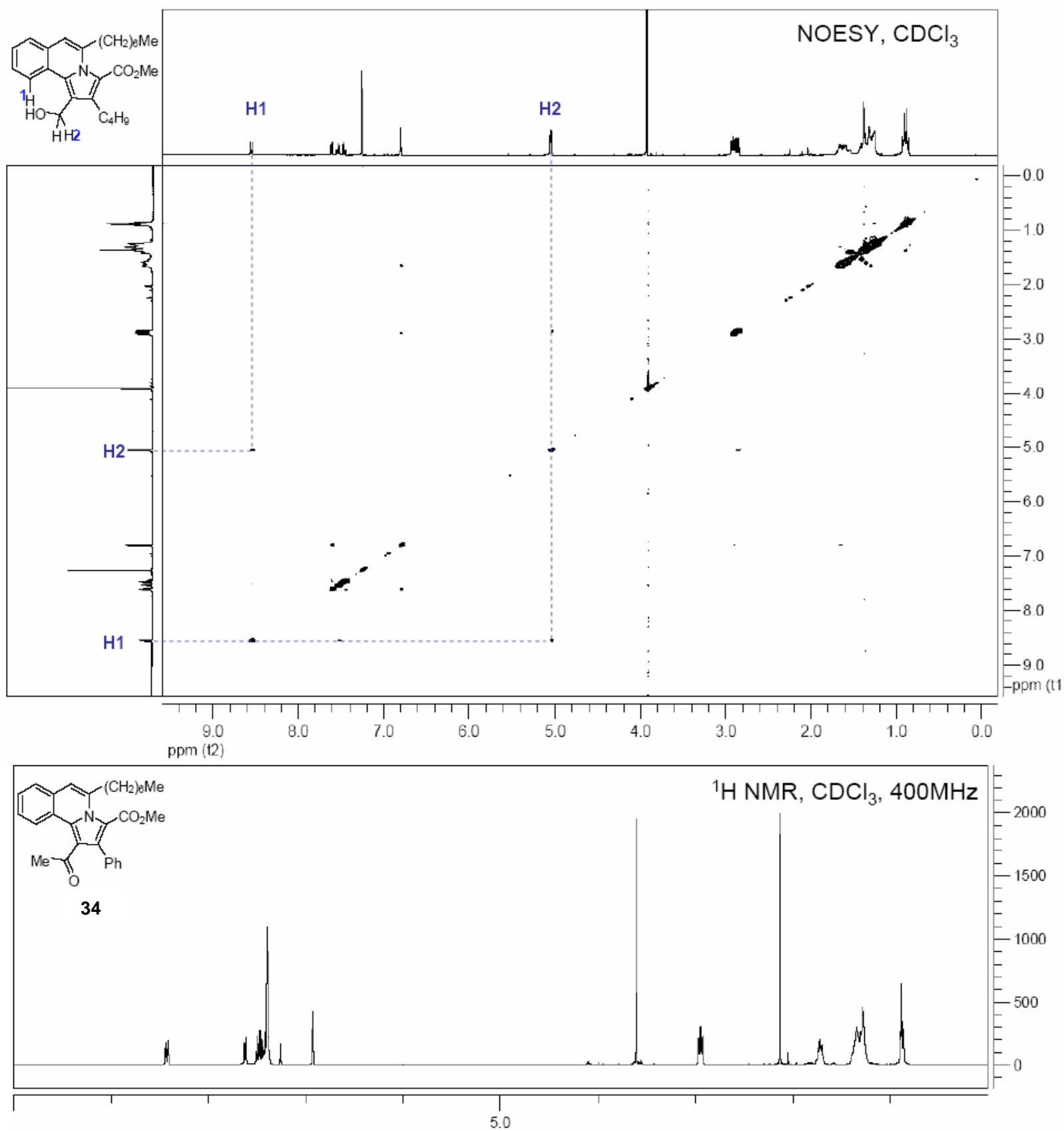

ppm (t1)

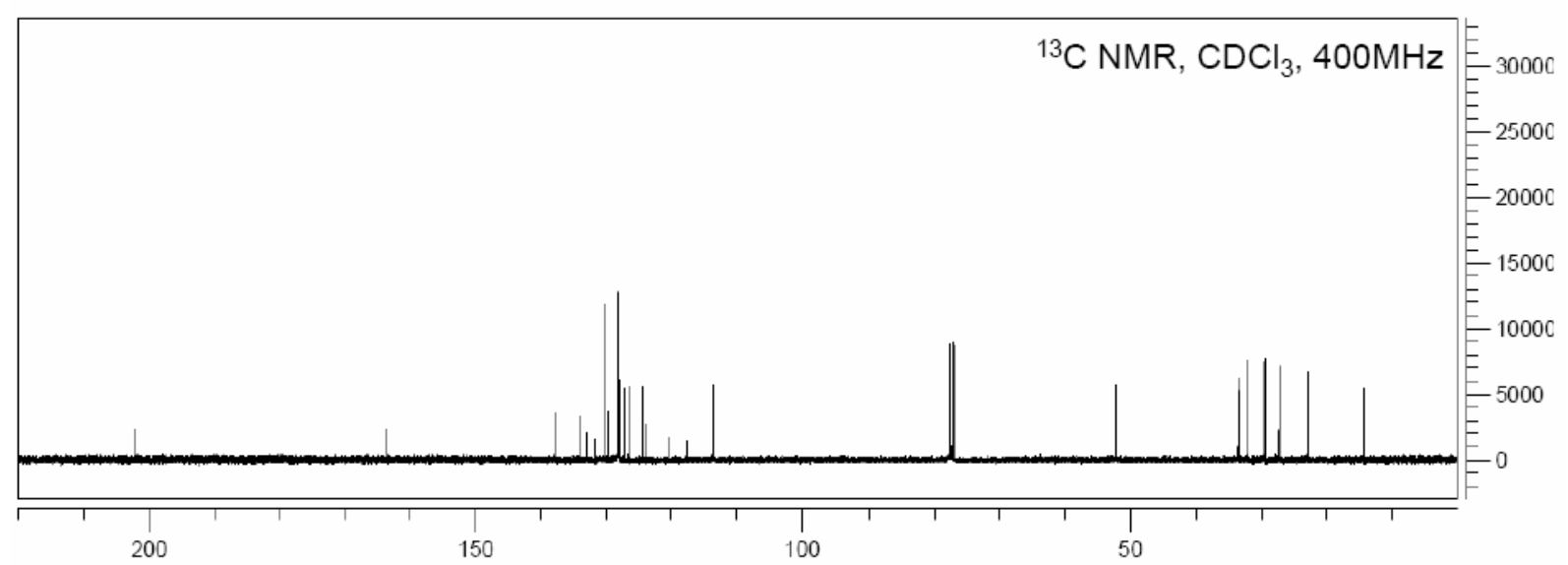
ppm (t1) 


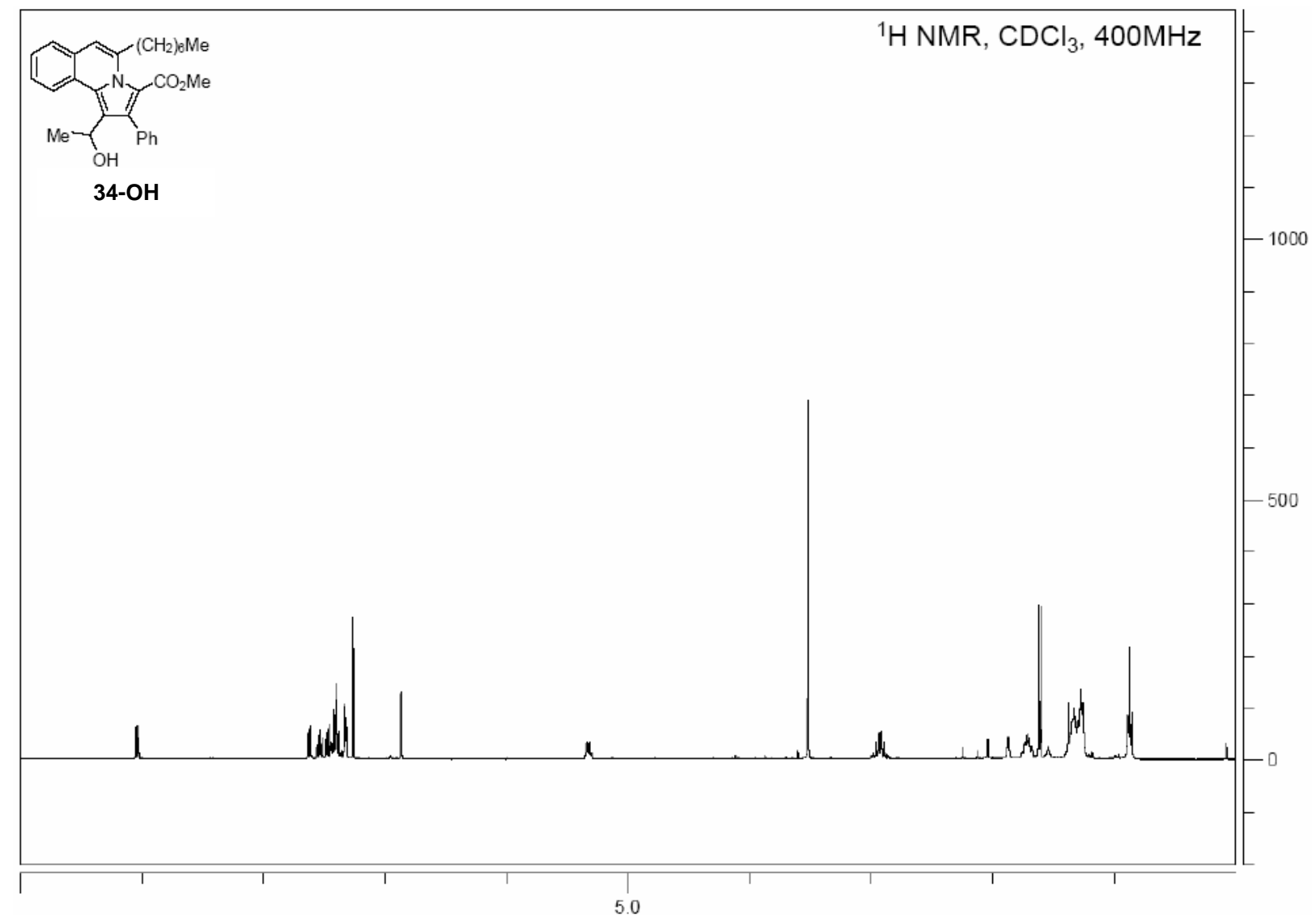

ppm (t1)

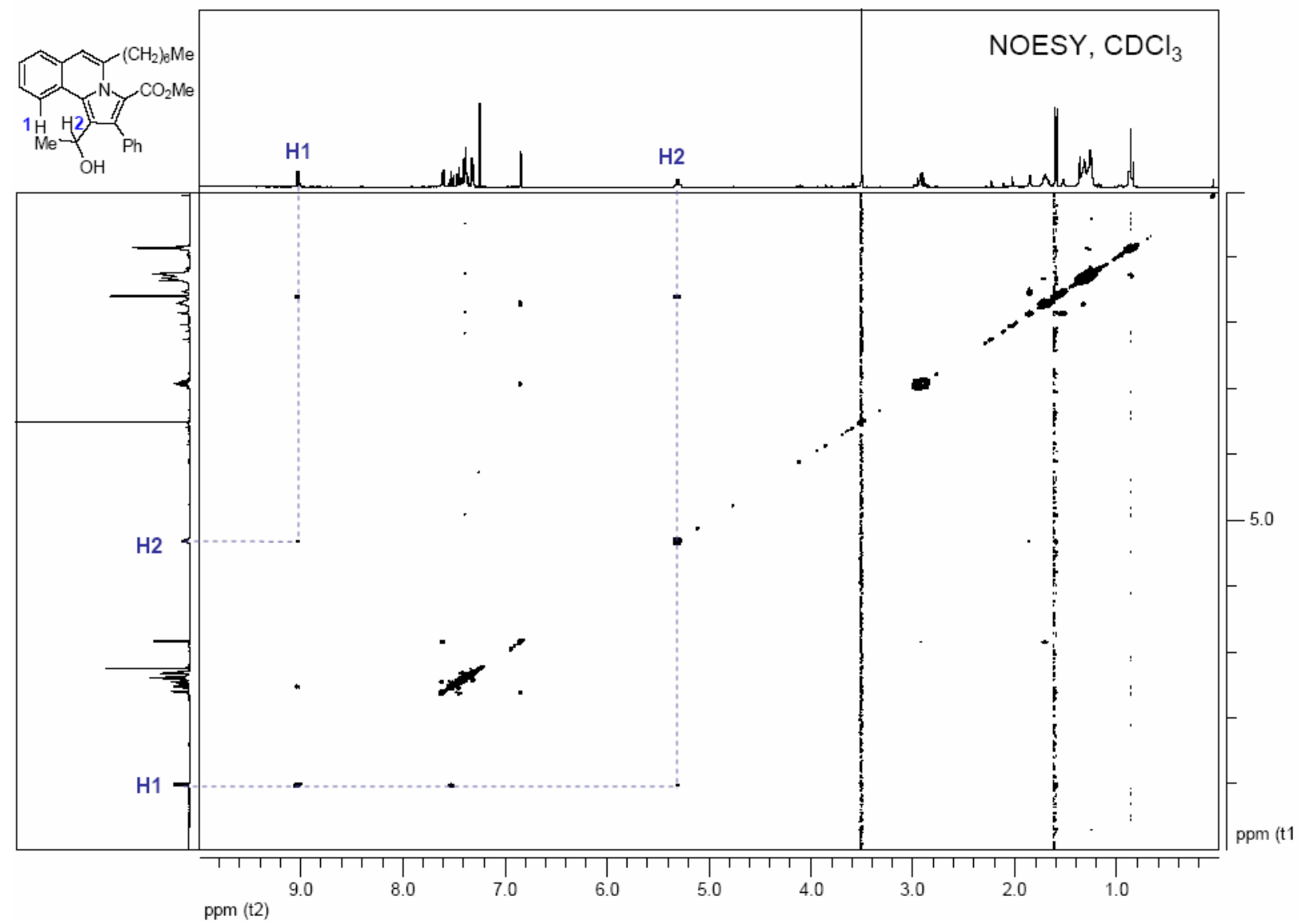




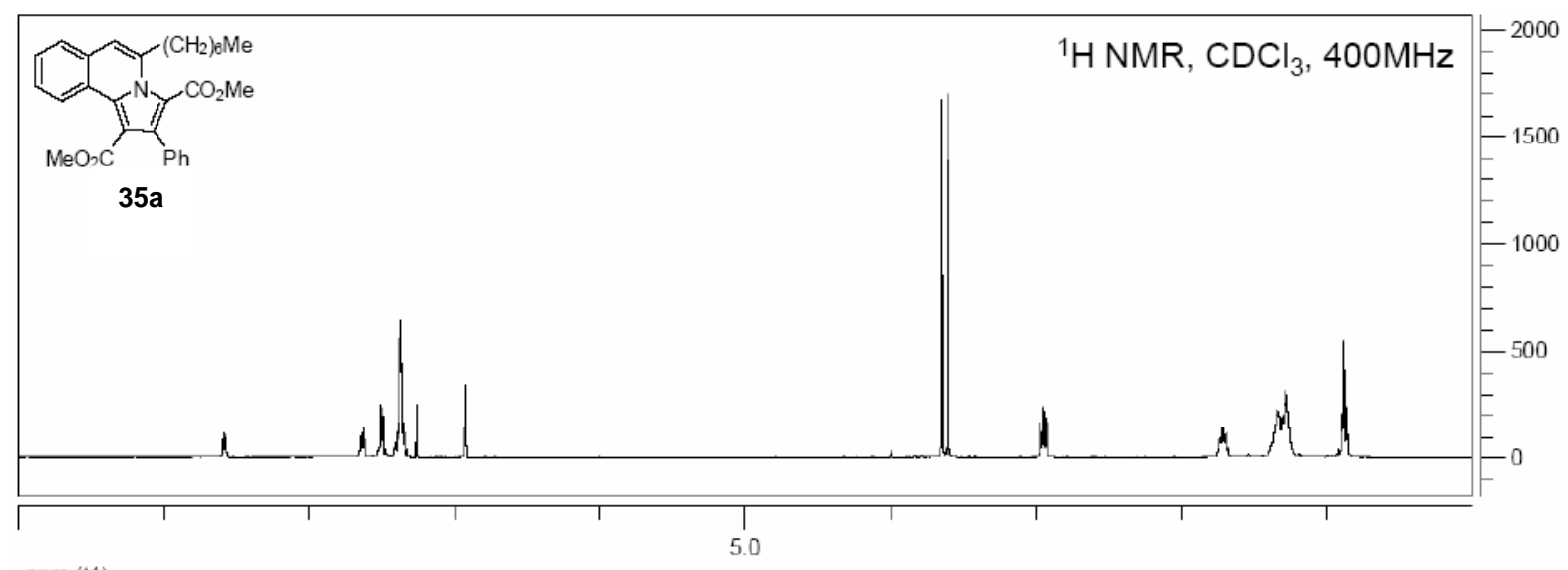

ppm (t1)
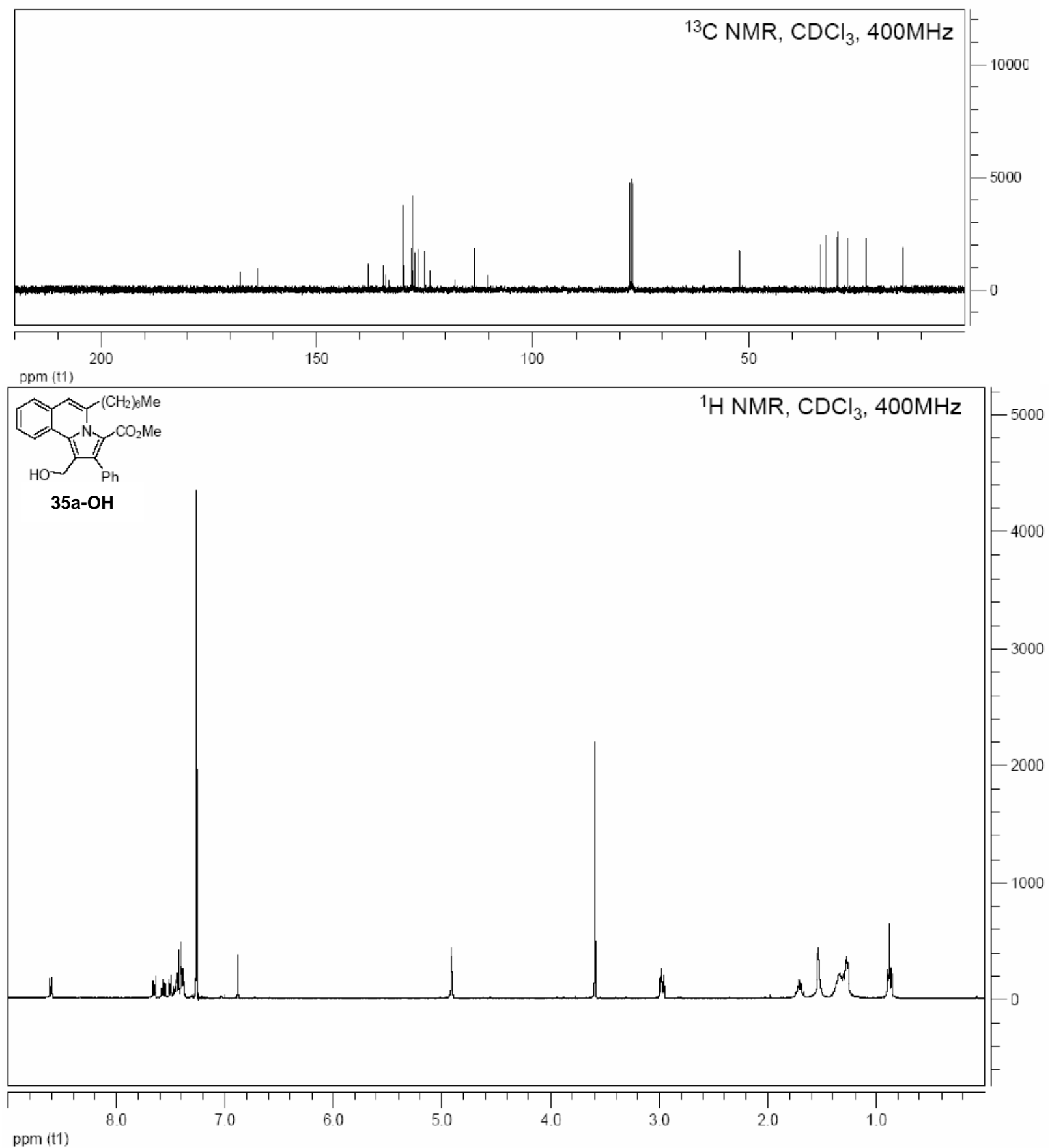

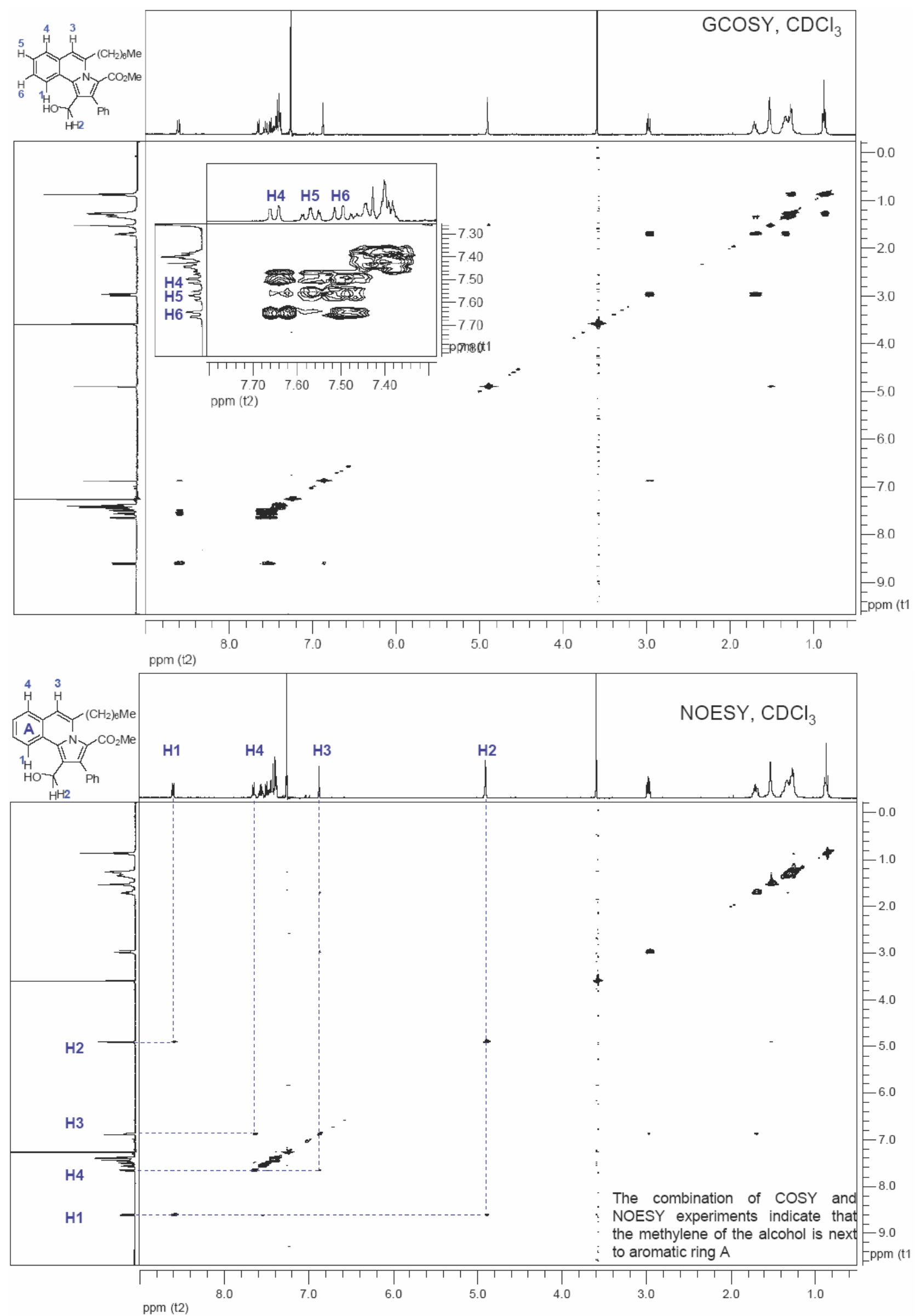


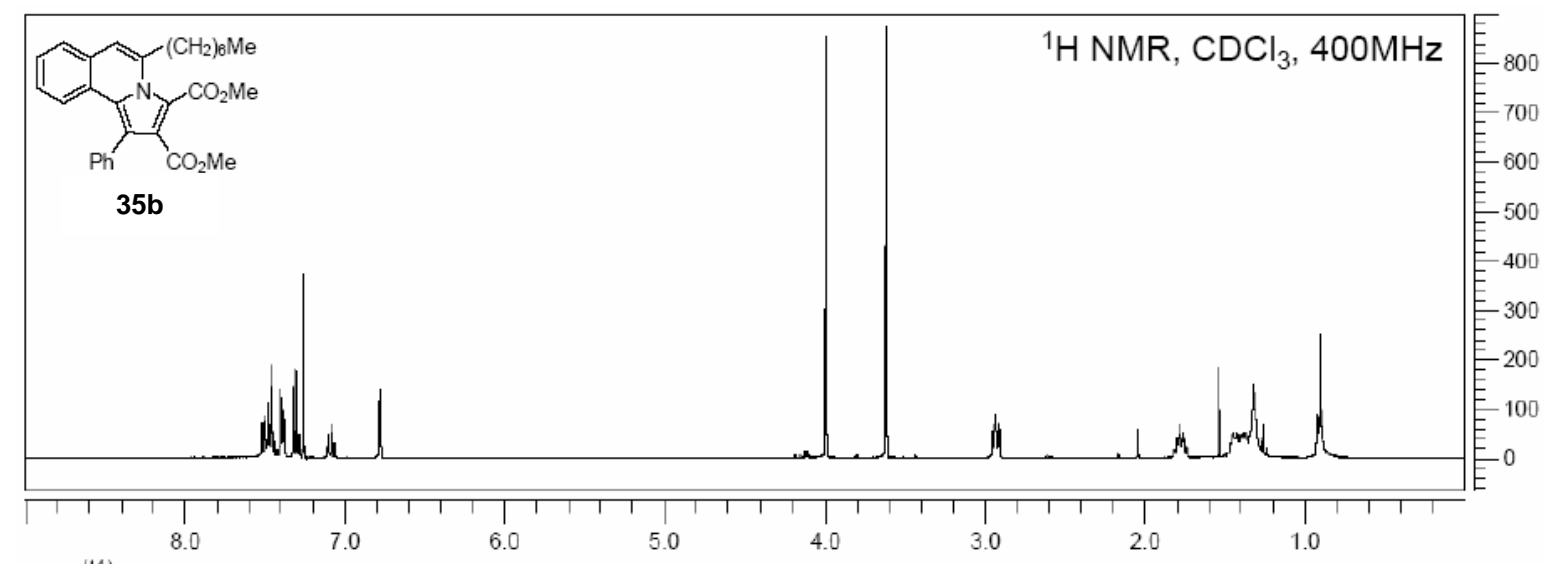

ppm (t1)
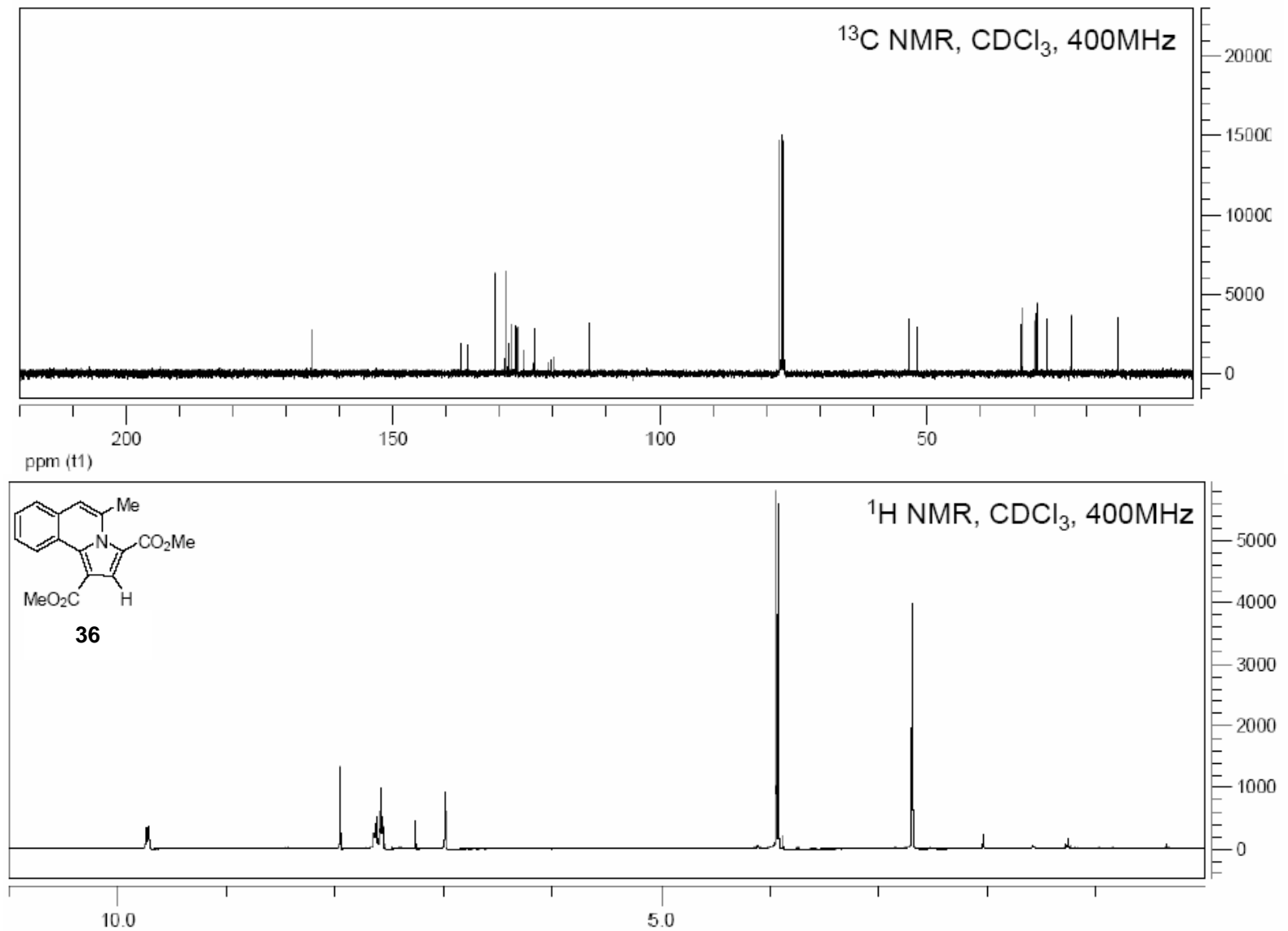

ppm (t1)

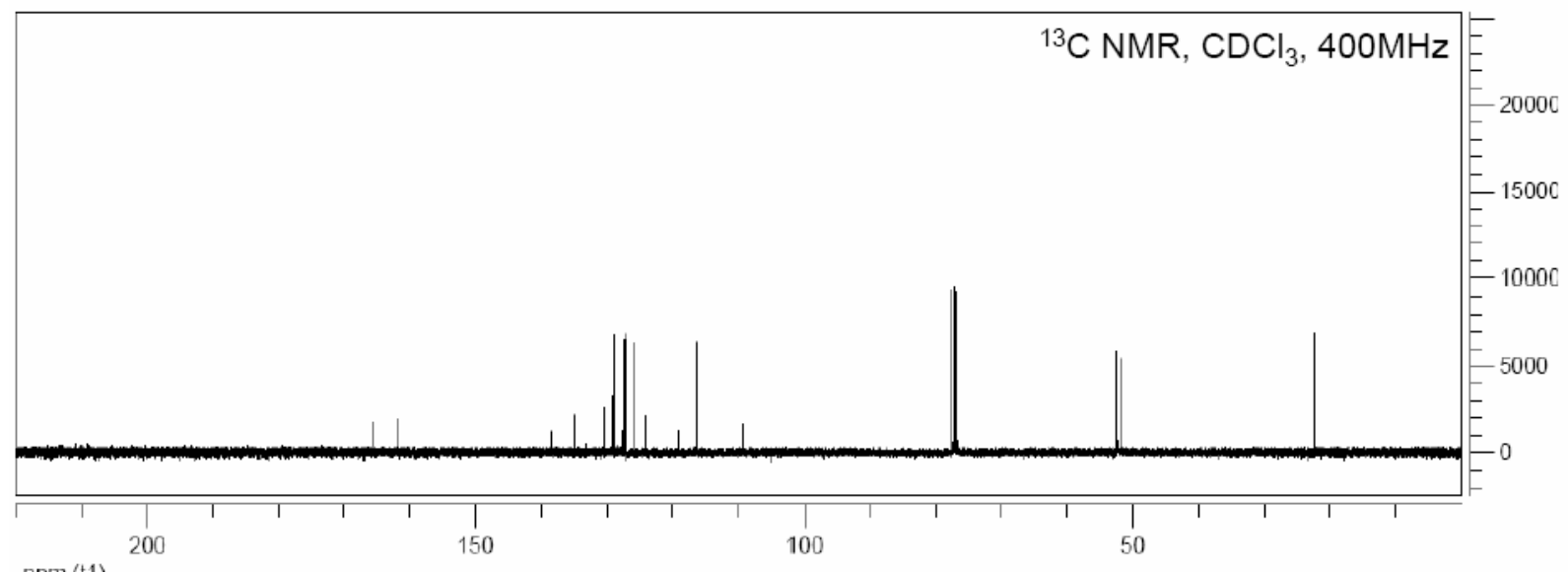

ppm (t1) 


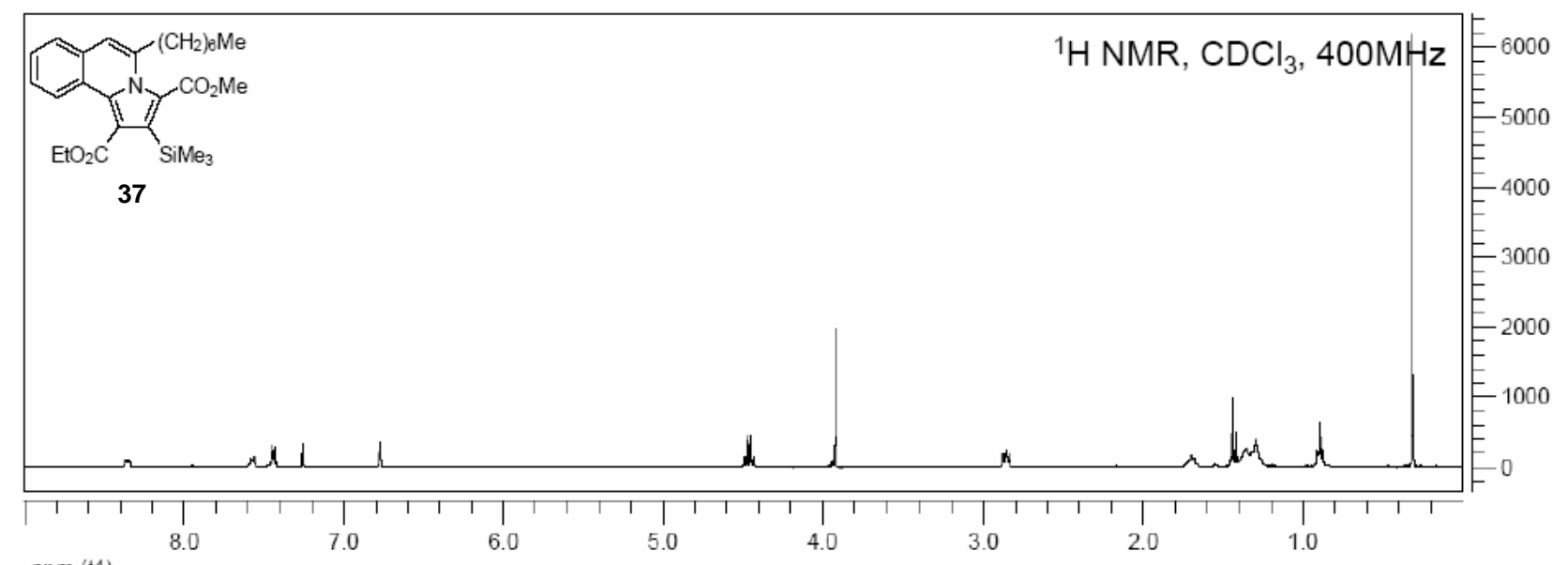

ppm (t1)

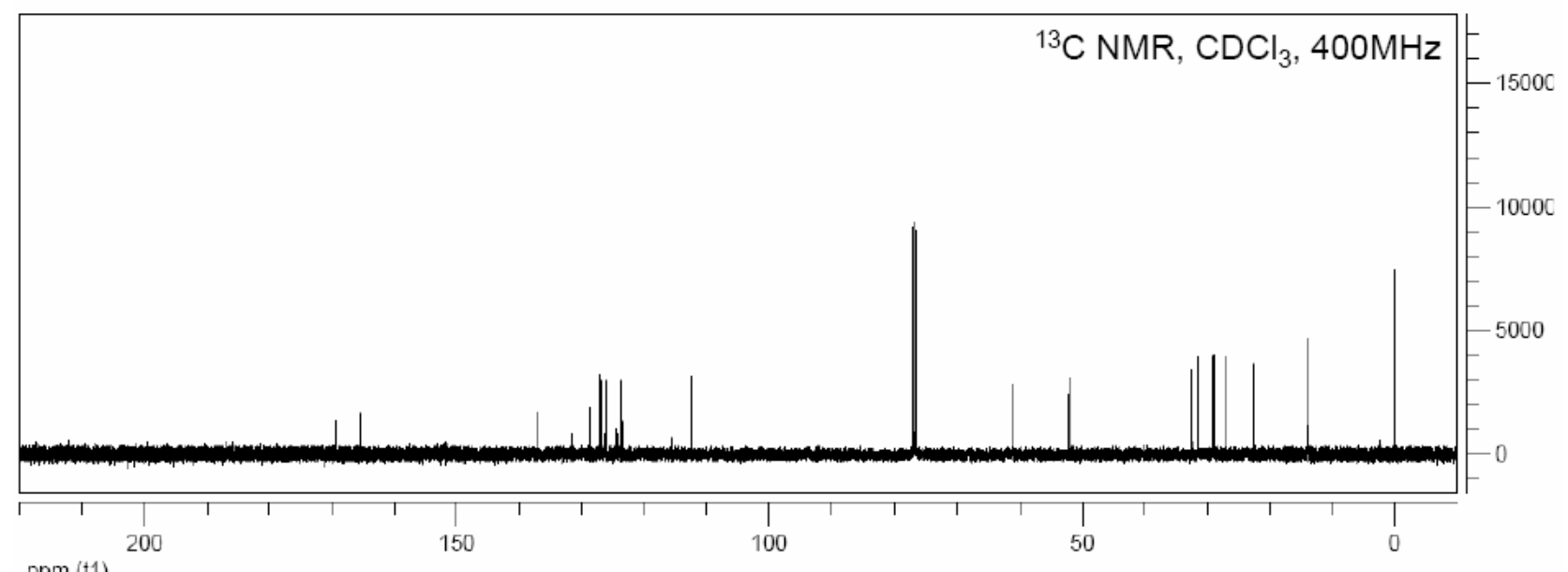

ppm (t1) 